\title{
Non-Commutative Integration of the Dirac Equation in Homogeneous Spaces
}

\author{
Alexander Breev ${ }^{1, *,+}$ (D) and Alexander Shapovalov $1,2,+(\mathbb{D}$ \\ 1 Department of Theoretical Physics, Tomsk State University, 1 Novosobornaya Sq., 634050 Tomsk, Russia; \\ shpv@phys.tsu.ru \\ 2 Department of Mathematics and Informatics, Tomsk Polytechnic University, 30 Lenin ave., \\ 634034 Tomsk, Russia \\ * Correspondence: breev@mail.tsu.ru \\ + These authors contributed equally to this work.
}

Received: 25 October 2020; Accepted: 12 November 2020; Published: 13 November 2020

check for updates

\begin{abstract}
We develop a non-commutative integration method for the Dirac equation in homogeneous spaces. The Dirac equation with an invariant metric is shown to be equivalent to a system of equations on a Lie group of transformations of a homogeneous space. This allows us to effectively apply the non-commutative integration method of linear partial differential equations on Lie groups. This method differs from the well-known method of separation of variables and to some extent can often supplement it. The general structure of the method developed is illustrated with an example of a homogeneous space which does not admit separation of variables in the Dirac equation. However, the basis of exact solutions to the Dirac equation is constructed explicitly by the non-commutative integration method. In addition, we construct a complete set of new exact solutions to the Dirac equation in the three-dimensional de Sitter space-time $\mathrm{AdS}_{3}$ using the method developed. The solutions obtained are found in terms of elementary functions, which is characteristic of the non-commutative integration method.
\end{abstract}

Keywords: Dirac equation; non-commutative integration; homogeneous spaces; induced representations; orbit method

\section{Introduction}

Exact solutions of the relativistic wave equations in strong gravitational and electromagnetic fields are the basis for studying quantum effects in the framework of quantum field theory in curved space-time (see, e.g., [1-6]). A construction of the complete set of exact solutions to these equations in many cases is associated with the presence of integrals of motion. For example, to separate the variables in a wave equation, it is necessary to have $\operatorname{dim} M-1$ commuting integrals, where $M$ is the space of independent variables. In this paper, by integrability of the wave equation, we mean an explicit possibility of reducing the original equation to a system of ordinary differential equations, the solution of which provides a complete set of solutions to the original wave equation.

The best-known technique for such a reduction is based on the method of separation of variables (SoV) (various aspects of the SoV method can be found, e.g., in [7-9]). There is a broad scope of research dealing with separation of variables in relativistic quantum wave equations, mainly for the Klein-Gordon and Dirac equations, and with classification of external fields admitting SoV in these equations (see, e.g., [10] and references therein). This motivates the development of methods for the exact integration of wave equations other than $\mathrm{SoV}$ that can give some new possibilities in relativistic quantum theory. 
In this regard, we focus on homogeneous spaces as geometric objects with high symmetry. We also note that most of the physically interesting problems and effects are associated with gravitational fields possessing symmetries. Mathematically, these symmetries indicate the presence of various groups of transformations that leave invariant the gravitational field. Representing the space-time as a homogeneous space with a group-invariant metric, we can consider a large class of gravitational fields and cosmological models [11,12] with rich symmetries, and the corresponding relativistic equations in these fields have integrals of motion.

We note that the relativistic wave equations on a homogeneous space may not allow separation of variables. The matter is that, in accordance with the theorem of Refs. [13,14], for the separation of variables in the wave equation in an appropriate coordinate system, the equation should admit a complete set of mutually commuting symmetry operators (integrals of motion, details can be found in $[13,14]$, see also [15]). Therefore, the problem arises of constructing exact solutions to the wave equation in the case when it has symmetry operators, but they do not form a complete set and separation of variables can not be carried out. We consider the non-commutative integration method (NCIM) based on non-commutative algebras of symmetry operators admitted by the equation [16-20]. This method can be thought as a generalization of the method of SoV. A reduction of the wave equation to a system of ODEs according to the NCIM (we use the term non-commutative reduction) can be carried out in a way that is substantially different from the method of separation of variables.

We note that the method of non-commutative integration has shown its effectiveness in constructing bases of exact solutions to the Klein-Gordon and Dirac equations in some spaces with invariance groups.

For instance, the NCIM was applied to the Klein-Gordon equation in homogeneous spaces with an invariant metric in $[19,20]$. The polarization vacuum effect of a scalar field in a homogeneous space was studied using NCIM in [19-21].

The non-commutative reduction of the Dirac equation to a system of ordinary differential equations in the Riemannian and pseudo-Riemannian spaces with a nontrivial group of motions was considered in [22-27]. In Refs. [28,29], the NCIM was applied to the Dirac equation in the four-dimensional flat space and in the de Sitter space. The Dirac equation on Lie groups that can be a special case of homogeneous spaces with a trivial isotropy subgroup was explored in terms of the NCIM in Refs. [30,31].

It may also be worth noting that the application of the NCIM to the Dirac equation can give a new class of its exact solutions, different from the solutions obtained by SoV. In cases where the Dirac equation does not admit separation of variables, the NCIM provides an uncontested option for constructing complete sets of solutions. The physical meaning of the solutions obtained by this method depends on the specifics of the problem being solved and requires special research in each case.

In the present work, we consider non-commutative symmetries of the Dirac equation in homogeneous spaces. We also develop the method of non-commutative integration of the Dirac equation in homogeneous spaces. Using the group-theoretic approach, we reduce the Dirac equation on the homogeneous space to such a system of equations on the transformation group that lets us apply the non-commutative reduction and construct exact solutions of the Dirac equation. In this paper, for the first time, we explicitly take into account the identities for generators of the transformation group in the problem of non-commutative reduction for the Dirac equation.

The work is organized as follows. In Section 2, we briefly introduce basic concepts and notations from the theory of homogeneous spaces [32-34], in order to be used later.

A construction of invariant differential operator with matrix coefficients on a homogeneous space is introduced in Section 3 following Refs. [35,36]. In addition, in this section, we show the connection between generators of the representation of a Lie group on a homogeneous space and the other representation induced by representation of a subgroup, whose action on a homogeneous space has a stationary point. 
In the next Section 4, we introduce a special irreducible representation of the Lie algebra of the Lie group of transformations of a homogeneous space using the Kirillov orbit method [37] that is necessary for non-commutative reduction.

In Section 5, we present the Dirac equation in a homogeneous space with an invariant metric in terms of an invariant matrix operator of the first order. The spinor connection and symmetry operators of the Dirac equation are shown to define isotropy representation in a spinor space. Generators of the spinor representation are found explicitly.

We also introduce a system of differential equations on the Lie group of transformations of a homogeneous space, which is equivalent to the original Dirac equation in a homogeneous space.

Then, in Section 6, we present a non-commutative reduction of the Dirac equation on a homogeneous space, using the irreducible $\lambda$-representation introduced in Section 4 and functional relations between symmetry operators (identities) for the Dirac equation.

In Section 7, we consider a homogeneous space with an invariant metric that does not admit separation of variables for the Klein-Gordon and Dirac equations. In this case, a complete set of exact solutions of the Dirac equation is constructed using the non-commutative reduction (Section 6).

The next Section 8 is devoted to the Dirac equation in the $(2+1)$ anti-de Sitter $\mathrm{AdS}_{3}$-dimensional space. In this homogeneous space, there are identities between the generators of the representation of the group $S O(1,3)$ that is taken into account when the non-commutative reduction is applied. The Dirac equation admits separation of variables in the $\mathrm{AdS}_{3}$ space, but the separable solutions are expressed through the special functions and have a complex form. The NCIM, being applied to this problem, results in the other complete set of exact solutions to the Dirac equation in the $\mathrm{AdS}_{3}$ space and these solutions are presented in terms of elementary functions.

In Section 9, we give our conclusion remarks.

\section{Invariant Metric on a Homogeneous Space}

This section introduces some basic concepts and notations of the homogeneous space theory with an invariant metric.

Let $G$ be a simply connected real Lie group with a Lie algebra $\mathfrak{g}, M$ be a homogeneous space with right action of the group $G,(x, g) \rightarrow R_{g} x=x g \in M$ for $x \in M, g \in G$. For any $x \in M$, there exists an isotropic subgroup $H_{x} \in G$. Denote by $H=H_{x_{0}}$ a closed stabilizer of a point $x_{0} \in M$, and let $\mathfrak{h}$ be a Lie algebra of $H$. The homogeneous space $M$ is diffeomorphic to a quotient manifold $G / H$ of right cosets $[H g]$ of the Lie group $G$ by $H$.

A transformation group $G$ can be regarded as a principal bundle $(G, \pi, M, H)$ with a structure group $H$, a base $M$, and a canonical projection $\pi: G \rightarrow M, \pi(e)=x_{0}$, where $e$ is the identity element of $G$. An arbitrary point $g \in G$ can be represented uniquely as $g=h s(x)$, where $x=x_{0} g=\pi(g) \in M$, $h \in H$, and $s: M \rightarrow G$ is a local and smooth section of $G, \pi \circ s=\mathrm{id}$.

Differential of the canonical projection $\pi_{*}: T_{g} G \rightarrow T_{\pi(g)} M$ is a surjective map that allows any tangent vector $\tau \in T_{x} M$ on a homogeneous space to be represented as $\pi_{*} \zeta$, where $\zeta \in T_{g} G$ is a tangent vector on $G$.

In turn, a linear space of the Lie algebra $\mathfrak{g} \simeq T_{e} G$ is decomposed into a direct sum of subspaces $\mathfrak{g}=\mathfrak{h} \oplus \mathfrak{m}$, where $\mathfrak{m}=\pi_{*}(\mathfrak{g}) \simeq T_{x_{0}} M$ is a complement to $\mathfrak{h}=\mathbf{k e r} \pi_{*}(e)$, i.e., $X=X_{\mathfrak{m}}+X_{\mathfrak{h}}$ holds for any $X \in \mathfrak{g}$, where $X_{\mathfrak{m}}=\pi_{*} X, X_{\mathfrak{h}} \in \mathfrak{h}$.

We introduce an invariant metric on the homogeneous space $M$. Let $\langle\cdot, \cdot\rangle_{\mathfrak{m}}$ be a non-degenerate $A d(H)$ - invariant scalar product on the subspace $\mathfrak{m}$,

$$
\left\langle[X, Y]_{\mathfrak{m}}, Z_{\mathfrak{m}}\right\rangle_{\mathfrak{m}}+\left\langle Y_{\mathfrak{m}},[X, Z]_{\mathfrak{m}}\right\rangle_{\mathfrak{m}}=0, \quad X \in \mathfrak{h}, \quad Y, Z \in \mathfrak{g}
$$


By action of a Lie group $G$ with right shifts on the homogeneous space $M$, we define the inner product throughout the space $M$ as

$$
\langle u, v\rangle_{x}=\left\langle\left(R_{g^{-1}}\right)_{*} u,\left(R_{g^{-1}}\right)_{*} v\right\rangle_{\mathfrak{m}}, \quad u, v \in T_{x} M, \quad x=\pi(g) .
$$

The $A d(H)$-invariance (1) is necessary and sufficient for the inner product (2) to be invariant with respect to the action of $G$ on $M$. The inner product defines an invariant metric on the homogeneous space $M$ [33].

On the principal bundle $(G, \pi, M, H)$, we introduce local coordinates $\left(x^{i}, h^{\alpha}\right),(i=1, \ldots, \operatorname{dim} M$, $\alpha=1, \ldots, \operatorname{dim} H)$ of the direct product $\mathrm{U} \times H$, where $\left\{x^{i}\right\}$ are local coordinates in a domain $U \subset M$ of a trivialization covering $x_{0} \in U$ with local coordinates $\left\{h^{\alpha}\right\}$ in the subgroup $H, e_{H}=\{0\}$. Thus, the local coordinates of an element $g=h s(x)$ from some neighborhood of the identity $G_{e}$ can be represented as $g^{i}=x^{i}, g^{\alpha}=h^{\alpha}$. We choose a section $s: M \rightarrow G$ so that equalities $s^{a}(x)=x^{a}$ and $s^{\alpha}(x)=0$ hold over the domain $U$.

The tangent vectors

$$
e_{a}=\left.s_{*} \partial_{x^{a}}\right|_{x=x_{0}}, \quad e_{\alpha}=\left.\partial_{h^{\alpha}}\right|_{h=e_{H}}
$$

form a basis $\left\{e_{A}\right\}=\left\{e_{a}\right\} \cup\left\{e_{\alpha}\right\},(A=1, \ldots, \operatorname{dim} \mathfrak{g})$ of the Lie algebra $\mathfrak{g} \simeq T_{x_{0}} M \oplus T_{e_{H}} H$, where $\left\{e_{\alpha}\right\}$ is a basis of the Lie algebra $\mathfrak{h} \simeq T_{e_{H}} H$, and $\left\{e_{a}\right\}$ is a basis of the linear space $\mathfrak{m} \simeq T_{x_{0}} M$. In the conjugate space $\mathfrak{g}^{*} \simeq T_{e}^{*} G$, we introduce the dual basis $\left\{e^{A}\right\}$ by the condition $\left\langle e^{A}, e_{B}\right\rangle=\delta_{B}^{A}$, where $\delta_{B}^{A}$ is the Kronecker symbol. The right-invariant basis vector fields $\eta_{A}(g)=-\left(R_{g}\right)_{*} e_{A}$ and the corresponding right-invariant 1-forms $\sigma^{A}(g)=-\left(R_{g}\right)^{*} e^{A}$ in local coordinates $(x, h)$ have the form

$$
\begin{gathered}
\eta_{a}(x, h)=\eta_{a}^{i}(x, h) \partial_{x^{i}}+\eta_{a}^{\alpha}(x, h) \partial_{h^{\alpha}}, \quad \eta_{\alpha}(x, h)=\eta_{\alpha}^{\beta}(h) \partial_{h^{\beta},} \\
\sigma^{a}(x, h)=\sigma_{i}^{a}(x, h) d x^{i}, \quad \sigma^{\alpha}(x, h)=\sigma_{i}^{\alpha}(x, h) d x^{i}+\sigma_{\beta}^{\alpha}(h) d h^{\beta}, \quad \alpha, \beta=1, \ldots, H .
\end{gathered}
$$

Here, $\left(R_{g}\right)_{*,}\left(R_{g}\right)^{*}$ are differentials of the right shifts $R_{g}\left(g^{\prime}\right)=g g^{\prime}$ on the Lie group $G$.

The right-invariant vector fields $\eta_{A}$ satisfy the commutation relations $\left[\eta_{A}, \eta_{B}\right]=C_{A B}^{C} \eta_{C}$, while the right-invariant 1-forms $\sigma^{A}$ satisfy the Maurer-Cartan relations, $-2 d \sigma^{C}=C_{A B}^{C} \sigma^{A} \wedge \sigma^{B}$. Here, $C_{A B}^{A}=\left[e_{A}, e_{B}\right]^{C}$ are the structure constants of $\mathfrak{g}$ with indices $A, B, C=1, \ldots, \operatorname{dim} G$.

We consider an invariant metric on the homogeneous space $M$ using a coordinate system in a domain $U$ of trivialization. A symmetric non-degenerate square matrix $G_{a b}=\left\langle e_{a}, e_{b}\right\rangle_{\mathfrak{m}}$ in a subspace $\mathfrak{m} \subset \mathfrak{g}$ satisfies the $\operatorname{Ad}(H)$-condition

$$
\begin{gathered}
G_{a b} C_{c \alpha}^{a}+G_{a c} C_{b \alpha}^{a}=0, \\
a, b=1, \ldots, \operatorname{dim} M, \quad \alpha=1, \ldots, \operatorname{dim} H .
\end{gathered}
$$

The invariant metric tensor in local coordinates $\left\{x^{i}\right\}$ is written as [38]:

$$
g_{i j}(x)=G_{a b} \sigma_{i}^{a}\left(x, e_{H}\right) \sigma_{j}^{b}\left(x, e_{H}\right), \quad i, j=1, \ldots, \operatorname{dim} M .
$$

The contravariant components of the metric tensor are

$$
g^{i j}(x)=G^{a b} \eta_{a}^{i}\left(x, e_{H}\right) \eta_{b}^{j}\left(x, e_{H}\right), \quad G^{a b}=\left(G_{a b}\right)^{-1} .
$$

In what follows, we will need the Christoffel symbols of the Levi-Civita connection with respect to a $G$-invariant metric $g_{M}$ given by $[19,33]$

$$
\begin{aligned}
\Gamma_{j k}^{i}(x)= & \Gamma_{b c}^{a} \sigma_{j}^{b}\left(x, e_{H}\right) \sigma_{k}^{c}\left(x, e_{H}\right) \eta_{a}^{i}\left(x, e_{H}\right)- \\
& \sigma_{j}^{b}\left(x, e_{H}\right) \eta_{b, k}^{i}\left(x, e_{H}\right)- \\
& C_{b \alpha}^{a} \sigma_{j}^{b}\left(x, e_{H}\right) \sigma_{k}^{\alpha}\left(x, e_{H}\right) \eta_{a}^{i}\left(x, e_{H}\right) .
\end{aligned}
$$


Here, $i, j, k=1, \ldots, \operatorname{dim} M$, and $\Gamma_{b c}^{a}$ are determined by $G^{a b}$ of the quadratic form $G$ and the structure constants of the Lie algebra $\mathfrak{g}$,

$$
\Gamma_{b c}^{a}=-\frac{1}{2} C_{b c}^{a}-\frac{1}{2} G^{a d}\left[G_{e c} C_{b d}^{e}+G_{e b} C_{c d}^{e}\right] .
$$

Thus, in a homogeneous space with invariant metric, the Levi-Civita connection is defined by algebraic properties of the homogeneous space.

\section{Induced Representations and Invariant First-Order Differential Operator with Matrix Coefficients}

Consider algebraic conditions for an invariant first-order linear differential operator with matrix coefficients on a homogeneous space $M$. We follow Ref. [35] where a more general case of invariant linear matrix differential operator of the second-order was studied.

Denote by $C^{\infty}(M, V)$ and $C^{\infty}(G, V)$ the two spaces of functions that map a homogeneous space $M$ and a transformation group $G$, respectively, to a linear space $V$. The last one can be regarded as a representation space of the algebra $\mathfrak{g l}(V)$.

Functions on the homogeneous space $M$ can be considered as defined on a Lie group $G$, but invariant over the fibers $H$ of the bundle $G$ [33]. In our case, when the functions take values in a vector space $V$, the space $C^{\infty}(M, V)$ is isomorphic to a subspace of the function space

$$
\hat{\mathcal{F}}=\left\{\varphi \in C^{\infty}(G, V) \mid \varphi(h g)=U(h) \varphi(g), \quad h \in H\right\},
$$

where $U(h)$ is an exact representation of the isotropy group $H$ in $V$. For any function $\varphi \in \hat{\mathcal{F}}$, we have

$$
\varphi(g)=\varphi(h s(x))=U(h) \varphi(s(x)), \quad g=(x, h) .
$$

Then, we can identify $\varphi(s(x))$ with a function $\varphi \in C^{\infty}(M, V)$. Equation (7) gives an explicit form of the isomorphism $\hat{\mathcal{F}} \simeq C^{\infty}(M, V)$. Differentiating relation (7) with respect to $h^{\alpha}$ and assuming $h=e_{H}$, we obtain

$$
\left(\eta_{\alpha}+\Lambda_{\alpha}\right) \varphi(g)=0, \quad \Lambda_{\alpha}=\left.\frac{\partial U(h)}{\partial h^{\alpha}}\right|_{h=e_{H}}, \quad \alpha=1, \ldots, \operatorname{dim} H
$$

Here, $\Lambda_{\alpha}$ are representation operators of the algebra $\mathfrak{h}$ on the space $V$. Equation (8) is a consequence of the condition (7) in the definition of $\hat{\mathcal{F}}$. The isotropy subgroup $H$ is assumed to be connected. Then, the conditions (7) and (8) are equivalent.

From (8), we can see that a linear differential operator $R=R\left(g, \partial_{g}\right)$ leaves invariant the function space $\hat{\mathcal{F}}$, if

$$
\left(\eta_{\alpha}+\Lambda_{\alpha}\right) R\left(g, \partial_{g}\right) \varphi(g)=\left[\eta_{\alpha}+\Lambda_{\alpha}, R\left(g, \partial_{g}\right)\right] \varphi(g)=0, \quad \varphi \in \hat{\mathcal{F}} .
$$

Thus, the space $L(\hat{\mathcal{F}})$ of linear differential operators $R\left(g, \partial_{g}\right): \hat{\mathcal{F}} \rightarrow \hat{\mathcal{F}}$ consists of linear differential operators on $C^{\infty}(G, V)$ provided that

$$
\left.\left[\eta_{\alpha}+\Lambda_{\alpha}, R\left(g, \partial_{g}\right)\right]\right|_{\hat{\mathcal{F}}}=0 .
$$

Then, given relation (7), the action of $R\left(g, \partial_{g}\right) \in L(\hat{\mathcal{F}})$ on a function $\varphi(g)$ from the space $\hat{\mathcal{F}}$ is written as

$$
R\left(g, \partial_{g}\right) \varphi(g)=U(h)\left(U^{-1}(h) R U(h)\right) \varphi(s(x)) .
$$


Multiplying Equation (9) by $U^{-1}(h)$ and given $\eta_{\alpha} U(h)=-\Lambda_{\alpha} U(h)$, we obtain

$$
\begin{gathered}
U^{-1}(h)\left[\eta_{\alpha}+\Lambda_{\alpha}, R\left(g, \partial_{g}\right)\right] U(h) \varphi(s(x))=\left[\eta_{\alpha}, U^{-1}(h) R\left(g, \partial_{g}\right) U(h)\right] \varphi(s(x))= \\
\eta_{\alpha}\left(U^{-1}(h) R\left(g, \partial_{g}\right) U(h) \varphi(s(x))\right)=0 .
\end{gathered}
$$

From here, it follows that the operator $U^{-1}(h) R\left(g, \partial_{g}\right) U(h)$ is independent of $h$ and (11) can be written as

$$
\begin{aligned}
& R\left(g, \partial_{g}\right) \varphi(g)=U(h) R_{M}\left(x, \partial_{x}\right) \varphi(s(x)), \\
& \left.R_{M}\left(x, \partial_{x}\right) \equiv\left(U^{-1}(h) R\left(g, \partial_{g}\right) U(h)\right)\right|_{h=e_{H}}= \\
& R\left(g, \partial_{g}\right) U(h)||_{h=e_{H}} .
\end{aligned}
$$

That is, for any operator $R\left(g, \partial_{g}\right)$ of $L(\hat{\mathcal{F}})$, there exists an operator $R_{M}$ on the homogeneous space $M$ acting on functions of the space $C^{\infty}(M, V)$. We say that the operator $R_{M}\left(x, \partial_{x}\right)$ is the projection of the operator $R\left(g, \partial_{g}\right): R_{M}\left(x, \partial_{x}\right)=\widehat{\pi}_{*} R\left(g, \partial_{g}\right)$. For example, for a first-order linear differential operator

$$
R_{1}\left(g, \partial_{g}\right)=B^{a}(x, h) \partial_{x^{a}}+B^{\alpha}(x, h) \partial_{h^{\alpha}}+B(x, h)
$$

the projection acts as follows:

$$
R_{M}^{(1)}\left(x, \partial_{x}\right)=\widehat{\pi}_{*} R_{1}\left(g, \partial_{g}\right)=B^{a}\left(x, e_{H}\right) \partial_{x^{a}}+B^{\alpha}\left(x, e_{H}\right) \Lambda_{\alpha}+B\left(x, e_{H}\right)
$$

On the other hand, any linear differential operator $R_{M}$ defined on $C^{\infty}(M, V)$ corresponds to an operator

$$
R\left(g, \partial_{g}\right)=U(h) R_{M}\left(x, \partial_{x}\right) U^{-1}(h) \in L(\hat{\mathcal{F}}) .
$$

Thus, we have the isomorphism $L(\hat{\mathcal{F}}) \simeq L\left(C^{\infty}(M, V)\right)$ whose explicit form is given by (12).

Let $\xi_{X}(g)=\left(L_{g}\right)_{*} X$ be a left-invariant vector field on the Lie group $G$, where $\left(L_{g}\right)_{*}: T_{g^{\prime}} G \rightarrow T_{g g^{\prime}} G$ is the left shift differential $L_{g}\left(g^{\prime}\right)=g g^{\prime}$ on $G, X \in \mathfrak{g}$.

Since the left-invariant vector fields commute with right-invariant ones, the condition of projectivity (10) is fulfilled. Using (13), we find the corresponding operator on the homogeneous space as

$$
\begin{aligned}
& \widetilde{X}(x)=\hat{\pi}_{*} \xi_{X}(g)=X(x)+\xi_{X}^{\alpha}\left(x, e_{H}\right) \Lambda_{\alpha}, \quad X \in \mathfrak{g}, \\
& X(x)=\xi_{X}^{a}(x) \partial_{x^{a}}, \quad \xi_{X}^{a}(x)=\left.\frac{d}{d t}\left(x e^{t X}\right)^{a}\right|_{t=0},
\end{aligned}
$$

where $X(x)$ are the generators of the action of the group $G$ on $M$, note that $X(x)$ act in the space $C^{\infty}(M)$. It is easy to verify the following commutation relations for operators (14):

$$
\begin{gathered}
{[\tilde{X}, \tilde{Y}]=\left.\left[U^{-1}(h) \xi_{X} U(h), U^{-1} \xi_{Y} U(h)\right]\right|_{h=e_{H}}=} \\
=\left.\left[U^{-1}(h)\left[\xi_{X}, \xi_{Y}\right] U(h)\right]\right|_{h=e_{H}}=\left.\left[U^{-1}(h) \xi_{[X, Y]} U(h)\right]\right|_{h=e_{H}}=\widetilde{[X, Y]}
\end{gathered}
$$

for all $X, Y \in \mathfrak{g}$. Consequently, the operators $\widetilde{X}$ corresponding to the left-invariant vector fields $\tilde{\xi} X$ are generators of a transformation group acting on $C^{\infty}(M, V)$ and are a continuation of the vector fields $X(x)$ obeying the same commutation relations. We show how the projection of left-invariant vector fields is related to representations of the group on homogeneous space. The space $\hat{\mathcal{F}}$ invariant under right shifts on the Lie group $G$ and the operators $T_{g}$, acting by the rule $\left(T_{g} \varphi\right)\left(g^{\prime}\right)=\varphi\left(g g^{\prime}\right)$, 
define a representation of the group $G$ on $\hat{\mathcal{F}}$ induced by the representation $U(h)$ of the subgroup $H$. According to relation (7), we have:

$$
\left(T_{g} \varphi\right)(s(x))=\varphi(g s(x))=\varphi(h(x, g) s(x g))=U(h(x, g)) \varphi(s(x g)),
$$

where $h(x, g) \in H$ is the factor of the homogeneous space [37], which is determined from the system of equations

$$
s(x) g=h(x, g) s(x g), \quad h(x, e)=e_{H} .
$$

In view of the isomorphism $\hat{\mathcal{F}} \simeq C^{\infty}(M, V)$, we obtain from (16) a representation of the Lie group $G$ on the space of functions $\psi \in C^{\infty}(M, V)$,

$$
\left(T_{g} \psi\right)(x)=U(h(x, g)) \psi(x g) .
$$

This representation is called the induced representation of the group $G$ on the homogeneous space $M$. Note that

$$
\xi_{X}(g) h^{\alpha}(x, g)=\xi_{X}(g)(h(x, g) s(x g))^{\alpha}=\xi_{X}(g)(s(x) g)^{\alpha}=\xi_{X}^{\alpha}(s(x) g),
$$

from whence the expression for the derivative of the factor at the identity element

$$
\left.\frac{d}{d t} h^{\alpha}\left(x, e^{t X}\right)\right|_{t=0}=\xi_{X}^{\alpha}\left(x, e_{H}\right)
$$

immediately follows.

It is easy to see that the operators $\widetilde{X}(x)$, as described by (15) and (17), are differentials of the representation $T_{g}$ on the homogeneous space $M$ :

$$
\widetilde{X}(x) \psi(x)=\left.\frac{d}{d t}\left(T_{\exp (t X)} \psi\right)(x)\right|_{t=0} .
$$

Thus, the projection of left-invariant vector fields on the group gives the infinitesimal operators of the representation of $T_{g}$ induced by the representation $U(h)$ of the subgroup $H$.

An operator $R_{M}\left(x, \partial_{x}\right) \in L\left(C^{\infty}(M, V)\right)$ is invariant under the action of the Lie group of transformations, if $R_{M}\left(x, \partial_{x}\right)$ commutes with $\widetilde{X}$ :

$$
\begin{gathered}
{\left[R_{M}\left(x, \partial_{x}\right), \widetilde{X}\right]=\left.\left[U^{-1}(h) R U(h), U^{-1}(h) \xi_{X} U(h)\right]\right|_{h=e_{H}}=} \\
=\left.U^{-1}(h)\left[R\left(g, \partial_{g}\right), \xi_{X}\right] U(h)\right|_{h=e_{H}}=0 .
\end{gathered}
$$

It follows that the operator $R_{M}\left(x, \partial_{x}\right)$ is invariant with respect to the transformation group if and only if the corresponding operator $R\left(g, \partial_{g}\right) \in L(\hat{\mathcal{F}})$ commutes with the left-invariant vector fields:

$$
\left[R\left(g, \partial_{g}\right), \xi_{X}\right]=0, \quad X \in \mathfrak{g} .
$$

Let $R_{M}^{(1)}\left(x, \partial_{x}\right) \in C^{\infty}(M, V)$ be a linear differential operator of the first order, invariant with respect to the group action. By (18), this operator corresponds to a first-order polynomial of right-invariant vector fields:

$$
R_{(1)}\left(g, \partial_{g}\right)=B^{a} \eta_{a}(x, h)+B^{\alpha} \eta_{\alpha}(h)+\tilde{B} .
$$

As a result of the projection, the expression $B^{\alpha} \eta_{\alpha}(h)$ becomes constant $B^{\alpha} \Lambda_{\alpha}$, which can be eliminated in the operator $R_{M}^{(1)}\left(x, \partial_{x}\right)$ by changing the variable $B=\tilde{B}+B^{\alpha} \Lambda_{\alpha}$. Therefore, we can 
put $B^{\alpha}=0$ without loss of generality. If we substitute the operator $R_{(1)}\left(g, \partial_{g}\right)$ in the projectivity condition (10), then we obtain

$$
\begin{gathered}
{\left.\left[\eta_{\alpha}+\Lambda_{\alpha}, R_{(1)}\left(g, \partial_{g}\right)\right]\right|_{\hat{\mathcal{F}}}=\left(\left[b^{a}, \Lambda_{\alpha}\right] \eta_{a}+b^{a}\left[\eta_{a}, \eta_{\alpha}\right]+\left[B, \Lambda_{\alpha}\right]\right)_{\hat{\mathcal{F}}}=} \\
\left.\left(\left[B^{a}, \Lambda_{\alpha}\right]+B^{b} C_{b \alpha}^{a}\right) \eta_{a}\right|_{\hat{\mathcal{F}}}+\left[B, \Lambda_{\alpha}\right]-B^{a} C_{a \alpha}^{\beta} \Lambda_{\beta}=0 .
\end{gathered}
$$

In addition, we have a system of algebraic equations for the coefficients $B^{a}$ and $B$ :

$$
\begin{aligned}
& {\left[B^{a}, \Lambda_{\alpha}\right]+B^{b} C_{b \alpha}^{a}=0,} \\
& {\left[B, \Lambda_{\alpha}\right]-B^{a} C_{a \alpha}^{\beta} \Lambda_{\beta}=0 .}
\end{aligned}
$$

When Equations (19) and (20) are fulfilled, the projection of $R_{(1)}\left(g, \partial_{g}\right)$ on the homogeneous space results in the desired form of the invariant linear differential operator of the first order:

$$
R_{M}^{(1)}\left(x, \partial_{x}\right)=\hat{\pi}_{*} R_{(1)}\left(g, \partial_{g}\right)=B^{a} \eta_{a}^{i}\left(x, e_{H}\right) \partial_{x^{i}}+B^{a} \eta_{a}^{\alpha}\left(x, e_{H}\right) \Lambda_{\alpha}+B
$$

Thus, any linear differential operator of the first order acting on the functions of $C^{\infty}(M, V)$ and being invariant with respect to the action of the transformation group has the form (21) where the matrix coefficients $B^{a}$ and $B$ satisfy the algebraic system of Equations (19) and (20). The matrices $\Lambda_{\alpha}$ are generators of the isotropy subgroup $H$ in a linear space $V$.

\section{4. $\lambda$-Representation of a Lie Algebra}

In this section, we describe a special representation of the Lie algebra $\mathfrak{g}$ using the orbit method [37]. The direct and inverse Fourier transforms on the Lie group $G$ are introduced that in what follows are necessary for the non-commutative reduction of the Dirac equation on the homogeneous space $M$. Here, we also use some results of the previous section.

First, we describe an orbit classification for the coadjoint representation of Lie groups following conventions of Refs. [39,40].

A degenerate Poisson-Lie bracket,

$$
\{\phi, \psi\}(f)=\langle f,[d \phi(f), d \psi(f)]\rangle=C_{A B}^{C} f_{C} \frac{\partial \phi(f)}{\partial f_{A}} \frac{\partial \psi(f)}{\partial f_{B}}, \quad \phi, \psi \in C^{\infty}\left(\mathfrak{g}^{*}\right),
$$

endows the space $\mathfrak{g}^{*}$ with a Poisson structure. Here, $f_{A}$ are coordinates of a linear functional $f=f_{A} e^{A} \in \mathfrak{g}^{*}$ relative to the dual basis $\left\{e^{A}\right\}$. The number ind $\mathfrak{g}$ of functionally independent Casimir functions $K_{\mu}(f)$ relative to the bracket (22) is called the index of the Lie algebra $\mathfrak{g}$.

A coadjoint representation on $\mathfrak{g}^{*}, \mathrm{Ad}^{*}: G \times \mathfrak{g}^{*} \rightarrow \mathfrak{g}^{*}$, stratifies $\mathfrak{g}^{*}$ into orbits of the coadjoint representation (K-orbits). The restriction of the bracket (22) on orbits is non-degenerate and coincides with the Poisson bracket generated by the symplectic Kirillov form $\omega_{\lambda}$.

The orbits of maximal dimension $\operatorname{dim} \mathcal{O}^{(0)}=\operatorname{dim} \mathfrak{g}-$ ind $\mathfrak{g}$ are called non-degenerate, and the those of less dimension are singular. We denote by $\mathcal{O}_{\lambda}^{(s)}$ the orbits of dimension $\operatorname{dim} \mathfrak{g}-\operatorname{ind} \mathfrak{g}-2 s$, $s=0, \ldots,(\operatorname{dim} \mathfrak{g}-$ ind $\mathfrak{g}) / 2$ passing through the functional $\lambda \in \mathfrak{g}^{*}$, and a number $s$ is called the orbit singularity index. A tangent space $T_{f} \mathcal{O}_{\lambda^{(s)}}$ to the orbit $\mathcal{O}_{\geq}{ }^{(s)}$ at a point $f$ is the linear span of vector fields

$$
Y_{A}(f)=C_{A B}(f) \frac{\partial}{\partial f_{B}}, \quad C_{A B}(f)=C_{A B}^{C} f_{C},
$$

so that the orbit dimension is given by the rank of the matrix $C_{A B}(f)$. The rank takes a constant value on the orbit, $\operatorname{dim} \mathcal{O}_{\lambda}^{(s)}=\operatorname{rank} C_{A B}(\lambda)$. The Kirillov form on tangent vectors to the orbit $\mathcal{O}_{\lambda}^{(s)}$ is $\omega_{\lambda}\left(Y_{A}, Y_{B}\right)=\left\langle\lambda,\left[Y_{A}, Y_{B}\right]\right\rangle$. 
The space $\mathfrak{g}^{*}$ can be decomposed into a sum of disjoint invariant algebraic surfaces $M_{s}$ consisting of orbits of the same dimension $\operatorname{dim} \mathfrak{g}-$ ind $\mathfrak{g}-2 s$ :

$$
\begin{aligned}
& M_{0}=\left\{f \in \mathfrak{g}^{*} \mid \neg\left(F^{1}(f)=0\right)\right\}, \\
& M_{s}=\left\{f \in \mathfrak{g}^{*} \mid F^{s}(f)=0, \neg\left(F^{s+1}(f)=0\right)\right\}, \quad s=1, \ldots, \frac{\operatorname{dim} \mathfrak{g}-\text { ind } \mathfrak{g}}{2}-1, \\
& M_{(\operatorname{dim} \mathfrak{g}-\text {-ind } \mathfrak{g}) / 2}=\left\{f \in \mathfrak{g}^{*} \mid F^{\frac{\text { dimg-ind }}{2}}(f)=0\right\},
\end{aligned}
$$

where $F^{s}(f)$ denotes the set of all minors of the matrix $C_{A B}(f)=C_{A B}^{C} f_{C}$ of size dim $\mathfrak{g}-$ ind $\mathfrak{g}-$ $2 s+2$; the notation $F^{s}(f)=0$ implies that all the corresponding minors at the point $f$ vanish, and $\neg\left(F^{S}(f)=0\right)$ means that, at the point $f$, the corresponding minors do not vanish simultaneously. In the general case, the surface $M_{S}$ is disconnected.

In what follows, by $M_{(s)}$, we denote a connected component of the surface $M_{s}$ containing the orbit $\mathcal{O}^{(s)}$, and $\mathcal{O}^{(s)}$ will be called the (s)-type orbit. Each component of $M_{(s)}$ is uniquely determined by a set of homogeneous polynomials $F_{\alpha}^{(s)}(f)$ satisfying the system

$$
\left.\Upsilon_{A}(f) F_{\alpha}^{(s)}(f)\right|_{F^{(s)}(f)=0}=0 .
$$

The non-constant functions $K_{\mu}^{(s)}(f)$ on $M_{(s)}$ are called the (s)-type Casimir functions if they commute with any function on $M_{(s)}$ with respect to the bracket (22). In other words, the functions $K_{\mu}^{(s)}(f)$ are invariants of the adjoint representation and are determined by the system:

$$
\left.Y_{A}(f) K_{\mu}^{(s)}(f)\right|_{f \in M_{(s)}}=0, \quad \mu=1, \ldots, r_{(s)} .
$$

The number of functionally independent solutions of this system is determined by the dimension of the surface $M_{(s)}$ :

$$
r_{(s)}=\operatorname{dim} M_{(s)}-(\operatorname{dim} \mathfrak{g}-\operatorname{ind} \mathfrak{g}-2 s) .
$$

Denote by $\Omega^{(s)} \subset \mathbb{R}^{r_{(s)}}$ a set of values of the mapping $K^{(s)}: M_{(s)} \rightarrow \mathbb{R}^{r_{(s)}}$ and introduce a locally invariant subset

$$
\mathcal{O}_{\omega}^{(s)}=\left\{f \in M_{(s)} \mid K_{\mu}^{(s)}(f)=\omega_{\mu}^{(s)}, \quad \mu=1, \ldots, r_{(s)}, \quad \omega^{(s)} \in \Omega^{(s)}\right\} .
$$

If the Casimir functions $K_{\mu}^{(s)}(f)$ are single-valued, then the level surface $\mathcal{O}_{\omega}^{(s)}$ consists of a countable set of orbits. We call $\mathcal{O}_{\omega}^{(s)}$ the class of orbits. As a result, the space $\mathfrak{g}^{*}$ consists of a union of connected invariant disjoint algebraic surfaces $M_{(s)}$, which in turn is the union of the orbit classes $\mathcal{O}_{\omega}^{(s)}$ :

$$
\mathfrak{g}^{*}=\bigcup_{(s)} M_{(s)}=\bigcup_{(s)} \bigcup_{\omega^{(s)} \in \Omega^{(s)}} \mathcal{O}_{\omega}^{(s)} .
$$

Consider a quotient space $B_{(s)}=M_{(s)} / G, \operatorname{dim} B_{(s)}=r_{(s)}$, whose points are the orbits of one class, $\mathcal{O}_{\lambda}^{(s)} \in M_{(s)}$. We introduce a local section $\lambda(j)$ of the bundle $M_{(s)}$ with base $B_{(s)}$ using real parameters $j=\left(j_{1}, \ldots, j_{r_{(s)}}\right)$ taking their values in a domain $J \subset \mathbb{R}^{r(s)}$ :

$$
F^{(s)}(\lambda(j))=0, \quad K_{\mu}^{(s)}(\lambda(j))=\omega_{\mu}^{(s)}(j), \quad \operatorname{det}\left\|\frac{\partial \omega_{\mu}^{(s)}(j)}{\partial j_{v}}\right\| \neq 0 .
$$


Let $\mathcal{O}_{\lambda(j)}^{(s)}$ be a K-orbit of (s)-type passing through a covector $\lambda=\lambda(j) \in \mathfrak{g}^{*}$ and belonging to the same class of orbits for all $j \in J$.

Using the Kirillov orbit method [37], we construct a unitary irreducible representation of the Lie group $G$ on a given orbit. This representation can be constructed if and only if for the functional $\lambda$ there exists a subalgebra $\mathfrak{p} \subset \mathfrak{g}^{\mathbb{C}}$ in the complex extension $\mathfrak{g}^{\mathbb{C}}$ of the Lie algebra $\mathfrak{g}$ satisfying the conditions:

$$
\langle\lambda,[\mathfrak{p}, \mathfrak{p}]\rangle=0, \quad \operatorname{dim} \mathfrak{p}=\operatorname{dim} \mathfrak{g}-\frac{1}{2} \operatorname{dim} \mathcal{O}_{\lambda}^{(s)} .
$$

The subalgebra $\mathfrak{p}$ is called the polarization of the functional $\lambda$. In (24), it is assumed that the functionals from the space $\mathfrak{g}^{*}$ are extended to $\mathfrak{g}^{\mathbb{C}}$ by linearity. Moreover, real polarizations always exist for nilpotent and completely solvable Lie algebras, and the complex polarizations always exist for solvable Lie groups [41]. For non-degenerate orbits $\mathcal{O}_{\lambda}^{(0)}$, there always exists, generally speaking, a complex polarization. In this paper, for simplicity, we restrict ourselves to the case when $\mathfrak{p}$ is the real polarization.

Denote by $P$ a closed subgroup of the Lie group $G$ whose Lie algebra is $\mathfrak{p}$. The Lie group acts on the right homogeneous space $Q \simeq G / P: q^{\prime}=q g$. According to the orbit method, we introduce a unitary one-dimensional irreducible representation of the Lie group $P$, which, in the neighborhood of the identity element of the group, has the form

$$
U^{\lambda}\left(e^{X}\right)=\exp \left(\frac{i}{\hbar}\langle\lambda, X\rangle\right), \quad X \in \mathfrak{p} .
$$

The representation of the Lie group $G$ corresponding to the orbit $\mathcal{O}_{\lambda}^{(s)}$ is induced using (25) as

$$
\begin{aligned}
& \left(T_{g}^{\lambda} \psi\right)(q)=\sqrt{\frac{\Delta_{G}(p(q, g))}{\Delta_{P}(p(q, g))}} U^{\lambda}(p(q, g)) \psi(q g)=U^{\lambda+i \hbar \beta}(p(q, g)) \psi(q g), \\
& \beta=\left.d \log \sqrt{\frac{\Delta_{G}(p)}{\Delta_{H}(p)}}\right|_{u=e_{P}},
\end{aligned}
$$

where $\Delta_{G}(g)=\operatorname{det}^{-1} A_{g}$ is the module of the Lie group $G, \Delta_{P}(p)=\operatorname{det} A d_{p}$ is the module of the subgroup $P, p \in P$, and $e_{P}$ is the identity element of $P$. A function $p(q, g)$ is the factor of the homogeneous space $Q$.

The functions $\psi^{\lambda}(q ; g)=\left(T_{g}^{\lambda} \psi\right)(q)$ on the group $G$ satisfy a condition similar to (7):

$$
\psi^{\lambda}(q ; p g)=U^{\lambda+i \hbar \beta}(p) \psi^{\lambda}(q ; g), \quad u \in P .
$$

The space of all such functions will be denoted by $\mathcal{F}^{\geq}$. Restriction of the left-invariant vector fields $\xi_{X}(g)$ to a homogeneous space $Q$, as follows from results of section 3 , is correctly defined, and the explicit form of the corresponding operator on the homogeneous space is given by (12):

$$
\begin{aligned}
& \ell_{X}\left(q, \partial_{q}, \lambda\right)=\left.\left(\left[U^{\lambda+i \hbar \beta}(p)\right]^{-1} \xi_{X}(g) U^{\lambda+i \hbar \beta}(p)\right)\right|_{p=e_{P}}, \\
& {\left[\ell_{X}\left(q, \partial_{q}, \lambda\right), \ell_{Y}\left(q, \partial_{q}, \lambda\right)\right]=\ell_{[X, Y]}\left(q, \partial_{q}, \lambda\right), \quad X, Y \in \mathfrak{g} .}
\end{aligned}
$$

Equation (27) shows that $\ell_{X}\left(q, \partial_{q}, \lambda\right)$ are infinitesimal operators of the induced representation (26),

$$
\ell_{X}\left(q, \partial_{q}, \lambda\right) \psi(q)=\left.\frac{d}{d t}\left(T_{\exp (t X)}^{\lambda} \psi\right)(q)\right|_{t=0} .
$$


Denote by $L(Q, \mathfrak{h}, \lambda)$ a space of functions on $Q$ where representation (26) is defined. The representation (26) is unitary with respect to a scalar product of the function space $L_{2}(Q, \mathfrak{h}, \lambda)$ :

$$
\left(\psi_{1}, \psi_{2}\right)=\int_{Q} \overline{\psi_{1}(q)} \psi_{2}(q) d \mu(q), \quad d \mu(q)=\rho(q) d q^{1} \ldots d q^{\operatorname{dim} Q} .
$$

The function $\rho(q)$ is determined from the Hermitian condition for the operators $-i \ell_{X}\left(q, \partial_{q}, \lambda\right)$ with respect to this scalar product (29).

The irreducible representation of the Lie algebra $\mathfrak{g}$ by the linear operators of the first order (27) dependent on $\operatorname{dim} \mathcal{O}_{\lambda}^{(s)} / 2$ variables is called $\lambda$ - representation of the Lie algebra $\mathfrak{g}$, and it was introduced in Ref. [16].

Let us describe a recipe for calculating operators of the $\lambda$-representation on the class of orbits $\mathcal{O}_{\omega}^{(s)}$. We find a local section $\lambda(j)$ of the bundle $M_{(s)}$ with the base $B_{(s)}$ for which there exists a polarization $\mathfrak{p}$ with a basis $\left\{e_{\bar{\alpha}}\right\}$. Let us then fix the basis $\left\{e_{\bar{a}}^{\prime}\right\}$ in the additional subspace $\mathfrak{m}=\mathfrak{p}^{\perp}$ to the subalgebra $\mathfrak{p}$. In a neighborhood of the identity element of the Lie group $G$, we introduce local coordinates of the second kind,

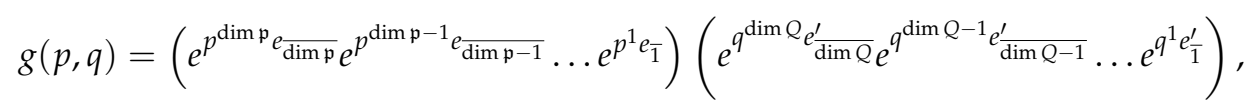

and write the left-invariant vector fields in local coordinates $(p, q)$ :

$$
\xi_{X}(p, q)=\xi_{X}^{\bar{a}}(q) \partial_{q^{\bar{a}}}+\xi_{X}^{\bar{\alpha}}(q, p) \partial_{p^{\bar{\alpha}}} .
$$

Next, operators of the $\lambda$ representation are defined using (27) as

$$
\begin{aligned}
& \ell_{X}\left(q, \partial_{q}, \lambda\right)=\xi_{X}^{\bar{\alpha}}(q) \partial_{q^{\bar{a}}}+\frac{i}{\hbar} \xi_{X}^{\bar{\alpha}}\left(q, e_{P}\right)\left(\lambda_{\bar{\alpha}}+i \hbar \beta_{\bar{\alpha}}\right), \\
& \beta_{\bar{\alpha}}=\frac{1}{2}\left[\operatorname{Tr}\left(\operatorname{ad}_{\bar{\alpha}}\right)-\operatorname{Tr}\left(\left.\operatorname{ad}_{\bar{\alpha}}\right|_{\mathfrak{p}}\right)\right] .
\end{aligned}
$$

In other words, finding of these operators is reduced to calculating the left-invariant vector fields on the group $G$ in the trivialization domain of the principal bundle of this group in the fibrations $P=\exp (\mathfrak{p})$.

Let us write the representation operators (26) in the integral form

$$
\begin{aligned}
& \left(T_{g}^{\lambda} \psi\right)(q)=\int_{Q} \psi\left(q^{\prime}\right) D_{q q^{\prime}}^{\lambda}(g) d \mu(q), \\
& D_{q q^{\prime}}^{\lambda}(g)=\sqrt{\frac{\Delta_{G}(p(q, g))}{\Delta_{H}(p(q, g))}} U^{\lambda}(p(q, g)) \delta\left(q g, q^{\prime}\right),
\end{aligned}
$$

where $\delta\left(q, q^{\prime}\right)$ is the generalized delta-function with respect to the measure $d \mu(q)$. The generalized kernels $D_{q q^{\prime}}^{\lambda}(g)$ of this representation satisfy the following properties:

$$
\begin{aligned}
& D_{q q^{\prime}}^{\lambda}\left(g_{1} g_{2}\right)=\int_{Q} D_{q q^{\prime \prime}}^{\lambda}\left(g_{1}\right) D_{q^{\prime \prime} q^{\prime}}^{\lambda}\left(g_{2}\right) d \mu\left(q^{\prime \prime}\right), \\
& D_{q q^{\prime}}^{\lambda}(g)=\overline{D_{q^{\prime} q}^{\lambda}\left(g^{-1}\right),} \quad D_{q q^{\prime}}^{\lambda}(e)=\delta\left(q, q^{\prime}\right),
\end{aligned}
$$

and the system of equations

$$
\begin{gathered}
\left(\eta_{X}(g)+\ell_{X}\left(q, \partial_{q}, \lambda\right)\right) D_{q q^{\prime}}^{\lambda}(g)=0 \\
\left(\xi_{X}(g)+\overline{\ell_{X}\left(q^{\prime}, \partial_{q^{\prime}}, \lambda\right)}\right) D_{q q^{\prime}}^{\lambda}(g)=0 .
\end{gathered}
$$


Here, $g_{1}, g_{2} \in G$. Let $G^{\lambda}=\left\{g \in G \mid A d_{g}^{*} \lambda=\lambda\right\}$ be a stabilizer of the functional $\lambda \in \mathfrak{g}^{*}$ with the Lie algebra $\mathfrak{g}^{\lambda}$, and the vectors $\left\{e_{\alpha}, \alpha=1, \ldots, \operatorname{dim} \mathfrak{g}^{\lambda}\right\}$ form some fixed basis for $\mathfrak{g}^{\lambda} \subset \mathfrak{p}$. As $P$ is the stabilizer of the point $q=0$ in the homogeneous space $Q$, and the group $G^{\lambda}$ lies in $P$, we get the equality

$$
\ell_{\alpha}\left(0, \partial_{q}, \lambda\right)=\frac{i}{\hbar}\left(\lambda_{\alpha}+i \hbar \beta_{\alpha}\right), \quad \alpha=1, \ldots, \operatorname{dim} \mathfrak{g}^{\lambda} .
$$

Restricting the first equality (31) to the subgroup $G^{\lambda}$ and setting $q=0$, we find

$$
\left(\eta_{\alpha}(h)+\frac{i}{\hbar}\left(\lambda_{\alpha}+i \hbar \beta_{\alpha}\right)\right) D_{0 q^{\prime}}^{\lambda}(h)=0, \quad h \in G^{\lambda} .
$$

The solution of the system (32) up to a constant factor can be represented as

$$
D_{0 q^{\prime}}^{\lambda}(h)=\exp \left(-\frac{i}{\hbar} \int \sigma^{\lambda}(h)+\int \sigma^{\bar{\alpha}}(h) \beta_{\bar{\alpha}}\right) .
$$

The subalgebra $\mathfrak{g}^{\lambda}$ is subordinate to the covector $\lambda$, and the 1 -forms $\sigma^{\lambda}(h)$ and $\sigma^{\bar{\alpha}}(h) \beta_{\bar{\alpha}}$ are closed in $G^{\lambda}$. Thus, the integral in (33) is well-defined. The local solution (33) can be extended to a global one, if the integral on the right-hand side of (33) over any closed curve $\Gamma$ on the subgroup $G^{\lambda}$ is a multiple of $2 \pi i$. Note that, since the 1 -form $\sigma^{\lambda}(h)$ is closed, the value of this integral depends only on the homological class to which the curve $\Gamma$ belongs. Therefore, for a global solution of the system (32), the following condition should be satisfied:

$$
\frac{1}{2 \pi \hbar} \oint_{\Gamma \in H_{1}\left(G^{\lambda}\right)} \sigma^{\lambda}(h)=n_{\Gamma} \in \mathbb{Z} .
$$

In other words, the 1-form $\sigma^{\lambda}(h)$ should belong to an integral cohomology class from $H^{1}\left(G^{\lambda}, \mathbb{Z}\right)$. In the case of a simply connected group $G$, the condition (34) is equivalent to the condition of integral orbit $\mathcal{O}_{\lambda}^{(s)}$, proposed by A. A. Kirillov [37]:

$$
\frac{1}{2 \pi \hbar} \int_{\Gamma \in H^{2}\left(\mathcal{O}_{\lambda}\right)} \omega_{\lambda}=n_{\Gamma} \in \mathbb{Z} .
$$

Thus, for a simply connected group, the coadjoint orbit $\mathcal{O}_{\lambda}^{(s)}$ is integral if the equality (34) is fulfilled.

A set of generalized functions $D_{q q^{\prime}}^{\lambda}(g)$ satisfying the system (31) was studied in Refs. [16,39], and the hypothesis was proposed that this set of generalized functions has the properties of completeness and orthogonality for a certain choice of the measure $d \mu(\lambda)$ in the parameter space $J$ :

$$
\begin{gathered}
\int_{G} \overline{D_{\widetilde{q} \widetilde{q}^{\prime}}^{\tilde{\lambda}}(g)} D_{q q^{\prime}}^{\lambda}(g) d \mu(g)=\Delta_{G}(s(q)) \delta(q, \tilde{q}) \delta\left(\tilde{q}^{\prime}, q^{\prime}\right) \delta(\tilde{\lambda}, \lambda), \\
\int_{Q \times Q \times J} \overline{D_{q q^{\prime}}^{\lambda}(\tilde{g})} D_{q q^{\prime}}^{\lambda}(g) d \mu(q) \frac{d \mu\left(q^{\prime}\right)}{\Delta_{G}(s(q))} d \mu(\lambda)=\delta(\tilde{g}, g) .
\end{gathered}
$$

Here, $\delta(g)$ is the generalized Dirac delta function with respect to the left Haar measure $d \mu(g)$ on the Lie group $G$.

For compact Lie groups, the relations (35) and (36) hold by the Peters-Weil theorem [42]. Note that, although there is no rigorous proof of the relations (35) and (36), in each case, it is easy to verify directly their validity. 
Consider the space $L(G, \lambda, d \mu(g))$ of functions of the form

$$
\psi^{\lambda}(g)=\int_{Q} \psi\left(q, q^{\prime}, \lambda\right) D_{q q^{\prime}}^{\lambda}\left(g^{-1}\right) d \mu\left(q^{\prime}\right) d \mu(q) .
$$

Here, a function $\psi\left(q, q^{\prime}, \lambda\right)$ of the two variables $q$ and $q^{\prime}$ belongs to the space $L(Q, \mathfrak{h}, \lambda)$. The inverse transform reads

$$
\psi\left(q, q^{\prime}, \lambda\right)=\Delta_{G}^{-1}\left(s\left(q^{\prime}\right)\right) \int_{Q \times Q \times J} \psi_{\lambda}(g) \overline{D_{q q^{\prime}}^{\lambda}\left(g^{-1}\right)} d \mu_{R}(g),
$$

where we have used (35) and (36), and $d \mu_{R}(g)=d \mu\left(g^{-1}\right)$ is the right Haar measure on the Lie group G.

The action of the operators $\xi_{X}(g)$ and $\eta_{X}(g)$ on the function $\psi^{\lambda}(g)$ from $L_{2}(G, \lambda, d \mu(g))$, according to (37) and (38), corresponds to action of the operators $\overline{\ell_{X}^{\dagger}\left(q, \partial_{q}, \lambda\right)}$ and $\ell_{X}\left(q^{\prime}, \partial_{q^{\prime}}, \lambda\right)$ on the function $\psi\left(q, q^{\prime}, \lambda\right)$, respectively,

$$
\begin{aligned}
& \xi_{X}(g) \psi^{\lambda}(g) \Longleftrightarrow \overline{\ell_{X}^{\dagger}\left(q, \partial_{q}, \lambda\right)} \psi\left(q, q^{\prime}, \lambda\right), \\
& \eta_{X}(g) \psi^{\lambda}(g) \Longleftrightarrow \ell_{X}\left(q^{\prime}, \partial_{q^{\prime}}, \lambda\right) \psi\left(q, q^{\prime}, \lambda\right) .
\end{aligned}
$$

The functions (37) are eigenfunctions for the Casimir operators $K_{\mu}^{(s)}(i \hbar \xi)=K_{\mu}^{(s)}(-i \hbar \eta)$. Indeed, from the system (31), we can obtain

$$
\begin{aligned}
& K_{\mu}^{(s)}(i \hbar \xi) \psi^{\lambda}(g)=K_{\mu}^{(s)}(-i \hbar \eta) \psi^{\lambda}(g)= \\
& \int_{Q}\left(\overline{K_{\mu}^{(s)}\left(-i \hbar \ell\left(q, \partial_{q}, \lambda\right)\right)} \psi\left(q, q^{\prime}, \lambda\right)\right) D_{q q^{\prime}}^{\lambda}\left(g^{-1}\right) d \mu\left(q^{\prime}\right)= \\
& \int_{Q}\left(K_{\mu}^{(s)}\left(-i \hbar \ell\left(q^{\prime}, \partial_{q^{\prime}}, \lambda\right)\right) \psi\left(q, q^{\prime}, \lambda\right)\right) D_{q q^{\prime}}^{\lambda}\left(g^{-1}\right) d \mu\left(q^{\prime}\right) .
\end{aligned}
$$

It follows that the operators $K_{\mu}^{(s)}\left(-i \hbar \ell\left(q^{\prime}, \lambda\right)\right)$ are independent of $q^{\prime}$ and

$$
\begin{aligned}
& K_{\mu}^{(s)}(i \hbar \xi) \psi^{\lambda}(g) \Longleftrightarrow \kappa_{\mu}^{(s)}(\lambda) \psi\left(q, q^{\prime}, \lambda\right), \\
& K_{\mu}^{(s)}\left(-i \hbar \ell\left(q^{\prime}, \partial_{q^{\prime}}, \lambda\right)\right)=\kappa_{\mu}^{(s)}(\lambda), \quad \overline{\kappa_{\mu}^{(s)}(\lambda)}=\kappa_{\mu}^{(s)}(\lambda), \\
& \lim _{\hbar \rightarrow 0} \kappa_{\mu}^{(s)}(\lambda)=\omega_{\mu}^{(s)}(\lambda) .
\end{aligned}
$$

Thus, as a result of the generalized Fourier transform (37), the left and the right fields become the operators of $\lambda$-representations, and the Casimir operators become constants. This fact is a key point for the method of non-commutative integration of linear partial differential equations on Lie groups, since it allows one to reduce the original differential equation with $\operatorname{dim} G$ independent variables to an equation with fewer independent variables equal to $\operatorname{dim} Q$.

\section{Dirac Equation in Homogeneous Space}

In this section, we consider the Dirac equation in an $n$-dimensional homogeneous space $M$ with an invariant metric. We shall assume that, in the homogeneous space $M$, an invariant metric $g_{M}$ and the Levi-Civita connection are given. Denote by $V_{\Psi}$ a space of spinor fields on $M$.

We write the Dirac equation in the space $M$ as an equation in an $n$-dimensional Lorentz manifold $M$ with the metric ( $\hbar$ is the Planck constant) as follows [43]:

$$
\left(i \hbar \gamma^{i}(x)\left[\nabla_{i}+\Gamma_{i}(x)\right]-m\right) \psi(x)=0, \quad i=1, \ldots, n .
$$


Here, $\nabla_{i}$ is the covariant derivative corresponding to the Levi-Civita connection on $M, m$ is mass of the field $\psi \in C^{\infty}\left(M, V_{\Psi}\right), \psi(x)$ is a column with $2^{\lfloor n / 2\rfloor}$ components, $\gamma^{i}(x)$ are $2^{\lfloor n / 2\rfloor} \times 2^{\lfloor n / 2\rfloor}$ gamma matrices,

$$
\left\{\gamma_{i}(x), \gamma_{j}(x)\right\}=2 g_{i j}(x) E, \quad i, j=1, \ldots, n,
$$

where $E$ denotes the $2^{\lfloor n / 2\rfloor} \times 2^{\lfloor n / 2\rfloor}$ identity matrix, $\Gamma_{i}(x)$ is the spinor connection satisfying the conditions $\left[\nabla_{i}, \gamma_{j}(x)\right]=0, \operatorname{Tr} \Gamma_{i}(x)=0$. The spinor connection $\Gamma_{i}(x)$ can be written as follows [43]:

$$
\Gamma_{i}(x)=-\frac{1}{4}\left(\nabla_{i} \gamma_{k}(x)\right) \gamma^{k}(x)
$$

We seek a solution of (40) with the decomposition

$$
\gamma^{i}(x)=\hat{\gamma}^{a} \eta_{a}^{i}\left(x, e_{H}\right), \quad \hat{\gamma}^{a}=\gamma^{i}(x) \sigma_{i}^{a}\left(x, e_{H}\right)
$$

The constant matrices $\hat{\gamma}^{a}$ satisfy the algebraic equations

$$
\left\{\hat{\gamma}^{a}, \hat{\gamma}^{b}\right\}=2 G^{a b} E, \quad a, b=1, \ldots, n .
$$

For the Dirac matrices with subscripts using (4), we have

$$
\gamma_{i}(x)=g_{i j}(x) \gamma^{i}(x)=\hat{\gamma}_{a} \sigma_{i}^{a}\left(x, e_{H}\right), \quad \hat{\gamma}_{b} \equiv G_{a b} \hat{\gamma}^{a} .
$$

The spinor connection is given by the following Lemma.

Lemma 1. The spinor connection $\Gamma(x)=\gamma^{i}(x) \Gamma_{i}(x)$ on the homogeneous space $M$ with invariant metric $g_{M}$ reads

$$
\begin{gathered}
\Gamma(x)=\hat{\gamma}^{a}\left(\Gamma_{a}+\eta_{a}^{\alpha}\left(x, e_{H}\right) \Lambda_{\alpha}\right), \quad \Gamma_{a}=-\frac{1}{4} \Gamma_{b a}^{d} \hat{\gamma}^{b} \hat{\gamma}_{d}, \\
\Lambda_{\alpha}=-\frac{1}{8} G_{a c} C_{\alpha b}^{a}\left[\hat{\gamma}^{b}, \hat{\gamma}^{c}\right] .
\end{gathered}
$$

Proof. The function $\Gamma(x)=\gamma^{i}(x) \Gamma_{i}(x)$ with $\Gamma_{i}(x)$ given by (41) can be written as

$$
\Gamma(x)=\frac{1}{4} \gamma^{i}(x) \gamma^{k}(x)\left(\partial_{x^{i}} \gamma_{k}(x)-\Gamma_{k i}^{l}(x) \gamma_{l}(x)\right),
$$

where $\Gamma_{k i}^{l}(x)$ are the Christoffel symbols, and $\partial_{x^{i}}$ is the partial derivative. Substituting (5), (42), and (44) in (46), we obtain

$$
\Gamma(x)=\Gamma+\frac{1}{4} C_{b \alpha}^{d} \hat{\gamma}^{a} \hat{\gamma}^{b} \hat{\gamma}_{d} \sigma_{i}^{\alpha}\left(x, e_{H}\right) \eta_{a}^{i}\left(x, e_{H}\right)
$$

Using property (3) of the invariant metric, we reduce the expression $C_{b \alpha}^{d} \hat{\gamma}^{b} \hat{\gamma}_{d}$ to the form

$$
C_{b \alpha}^{d} \hat{\gamma}^{b} \hat{\gamma}_{d}=C_{b \alpha}^{d} G_{d c} \hat{\gamma}^{b} \hat{\gamma}^{c}=-C_{c \alpha}^{d} G_{d b} \hat{\gamma}^{b} \hat{\gamma}^{c}=\frac{1}{2} C_{b \alpha}^{d} G_{d c}\left[\hat{\gamma}^{b}, \hat{\gamma}^{c}\right]=4 \Lambda_{\alpha} .
$$

From the chain of equalities

$$
\begin{gathered}
\sigma_{i}^{\alpha}\left(x, e_{H}\right) \eta_{a}^{i}\left(x, e_{H}\right)=\sigma_{A}^{\alpha}\left(x, e_{H}\right) \eta_{a}^{A}\left(x, e_{H}\right)-\sigma_{\beta}^{\alpha}\left(e_{H}\right) \eta_{a}^{\beta}\left(x, e_{H}\right)= \\
=\delta_{a}^{\alpha}-\left(-\delta_{\beta}^{\alpha}\right) \eta_{a}^{\beta}\left(x, e_{H}\right)=\eta_{a}^{\alpha}\left(x, e_{H}\right),
\end{gathered}
$$

we obtain for the spinor connection the required expression (45). 
Thus, the Dirac equation in the homogeneous space $M$ with an invariant metric $g_{M}$ and the Dirac matrices of the form (42) takes the form

$$
\begin{gathered}
\mathcal{D}_{M}\left(x, \partial_{x}\right) \psi=m \psi, \\
\mathcal{D}_{M}\left(x, \partial_{x}\right)=i \hbar \hat{\gamma}^{a}\left[\eta_{a}^{i}\left(x, e_{H}\right) \partial_{x^{i}}+\Gamma_{a}+\eta_{a}^{\alpha}\left(x, e_{H}\right) \Lambda_{\alpha}\right] .
\end{gathered}
$$

A set of matrices $\Lambda_{\alpha}$ determines a spinor representation of the isotopy subgroup $H$ in the space $V_{\Psi}$.

Lemma 2. The matrices $\Lambda_{\alpha}$ are generators of the isotropy subgroup $H$ representation on the space $V_{\Psi}$.

Proof. We prove that the matrices $\Lambda_{\alpha}$ satisfy the commutation relations:

$$
\left[\Lambda_{\alpha}, \Lambda_{\beta}\right]=C_{\alpha \beta}^{\gamma} \Lambda_{\gamma}
$$

The commutator of $\Lambda_{\alpha}$ and $\Lambda_{\beta}$ can be written as

$$
\left[\Lambda_{\alpha}, \Lambda_{\beta}\right]=-\frac{1}{4} C_{\beta b}^{d}\left[\Lambda_{\alpha}, \hat{\gamma}^{b} \hat{\gamma}_{d}\right]=-\frac{1}{4} C_{\beta b}^{d}\left(\left[\Lambda_{\alpha}, \hat{\gamma}^{b}\right] \hat{\gamma}_{d}+\hat{\gamma}^{b}\left[\Lambda_{\alpha}, \hat{\gamma}_{d}\right]\right)
$$

Using (3), (43) and (44), we find the commutator of $\Lambda_{\alpha}$ with $\hat{\gamma}^{a}$ :

$$
\begin{array}{r}
{\left[\Lambda_{\alpha}, \hat{\gamma}^{a}\right]=\frac{1}{4} C_{b \alpha}^{d}\left[\hat{\gamma}^{b} \hat{\gamma}_{d}, \hat{\gamma}^{a}\right]=\frac{1}{2} C_{b \alpha}^{d}\left(\delta_{d}^{a} \hat{\gamma}^{b}-G^{a b} \hat{\gamma}_{d}\right)=} \\
=\frac{1}{2}\left(G^{b d} C_{b \alpha}^{a}-G^{a b} C_{b \alpha}^{d}\right) \hat{\gamma}_{d}=C_{b \alpha}^{a} \hat{\gamma}^{b} .
\end{array}
$$

Similarly, for the $\gamma$-matrices with lower indices, we have

$$
\left[\Lambda_{\alpha}, \hat{\gamma}_{a}\right]=C_{\alpha a}^{b} \hat{\gamma}_{b}
$$

Substitution of (49) and (50) in (48) yields

$$
\left[\Lambda_{\alpha}, \Lambda_{\beta}\right]=\frac{1}{4}\left(C_{\beta e}^{d} C_{\beta b}^{e}-C_{\beta b}^{e} C_{\alpha e}^{d}\right) \hat{\gamma}^{b} \hat{\gamma}_{d}
$$

The expression inside the parentheses can be written in the form

$$
C_{\beta e}^{d} C_{\beta b}^{e}-C_{\beta b}^{e} C_{\alpha e}^{d}=\left[C_{\alpha b}^{A} C_{\beta A}^{d}+C_{b \beta}^{A} C_{\alpha A}^{d}+C_{\beta \alpha}^{A} C_{b A}^{d}\right]+C_{\alpha \beta}^{\gamma} C_{b \gamma}^{d} .
$$

By the Jacobi identity for the structure constants, the expression inside the square brackets is equal to zero. Substituting (52) in (51), we obtain (47).

The Dirac operator $\mathcal{D}_{M}(x)$ is a differential operator of the first order with matrix coefficients acting on the functions from $C^{\infty}\left(M, V_{\Psi}\right)$ (spinors). Let us associate this operator with the corresponding operator on the transformation group. The key theorem of our work is

Theorem 1. The Dirac operator $\mathcal{D}_{M}\left(x, \partial_{x}\right)$ on the homogeneous space $M$ with invariant metric $g_{M}$ is a projection of an operator

$$
\begin{gathered}
\mathcal{D}_{M}\left(x, \partial_{x}\right)=\hat{\pi}_{*} \mathcal{D}_{G}\left(g, \partial_{g}\right), \\
\mathcal{D}_{G}\left(g, \partial_{g}\right) \equiv i \hbar \hat{\gamma}^{a}\left[\eta_{a}(g)+\Gamma_{a}\right] \in L\left(\hat{\mathcal{F}}_{\Psi}\right) .
\end{gathered}
$$

Proof. Comparing the Dirac operator $\mathcal{D}_{M}\left(x, \partial_{x}\right)$ with invariant matrix differential operator of the first order (21) on the homogeneous space $M$, we obtain

$$
B^{a}=i \hbar \hat{\gamma}^{a}, \quad B=i \hbar \hat{\gamma}^{a} \Gamma_{a}
$$


Projection (53) is determined if the coefficients $B^{a}$ and $B$ of the form (54) satisfy Equations (19) and (20). From (49), it follows that the commutator of $\Lambda_{\alpha}$ and $\hat{\gamma}^{a}$ satisfies the first condition in (19). In this case, the condition (20) is reduced to

$$
\left[\Gamma, \Lambda_{\alpha}\right]=C_{a \alpha}^{\beta} \hat{\gamma}^{a} \Lambda_{\beta} .
$$

The commutator of $\Gamma$ and $\Lambda_{\alpha}$ can be presented in terms of the commutator $\left[\Lambda_{\alpha}, \Gamma_{a}\right]$ as

$$
\left[\Lambda_{\alpha}, \Gamma\right]=\left[\Lambda_{\alpha}, \hat{\gamma}^{a}\right] \Gamma_{a}+\hat{\gamma}^{a}\left[\Lambda_{\alpha}, \Gamma_{a}\right]
$$

Using (45) and (49) and (50), we get

$$
\begin{gathered}
{\left[\Lambda_{\alpha}, \Gamma_{a}\right]=-\frac{1}{4} \Gamma_{b a}^{d}\left(\left[\Lambda_{\alpha}, \hat{\gamma}^{b}\right] \hat{\gamma}_{d}+\hat{\gamma}^{b}\left[\Lambda_{\alpha}, \hat{\gamma}_{d}\right]\right)=} \\
\frac{1}{4} \Gamma_{b a}^{d}\left(C_{\alpha d}^{c} \hat{\gamma}^{b} \hat{\gamma}_{c}-C_{\alpha c}^{b} \hat{\gamma}^{c} \hat{\gamma}_{d}\right) .
\end{gathered}
$$

Substituting (57) into (56), we find

$$
\left[\Lambda_{\alpha}, \Gamma\right]=\frac{1}{4}\left(C_{\alpha a}^{e} \Gamma_{b e}^{c}-C_{\alpha c}^{c} \Gamma_{b a}^{e}+C_{\alpha b}^{e} \Gamma_{e a}^{c}\right) \hat{\gamma}^{a} \hat{\gamma}^{b} \hat{\gamma}_{c}
$$

From (6) and the Jacobi identity for structure constants of the Lie algebra $\mathfrak{g}$, it follows that

$$
C_{\alpha e}^{c} \Gamma_{b a}^{e}=C_{\alpha a}^{e} \Gamma_{b e}^{c}+C_{\alpha b}^{e} \Gamma_{e a}^{c}+C_{\alpha a}^{\beta} C_{\beta b}^{c} .
$$

Substituting (59) into (58), we obtain (55). Thus, relations (19) and (20) are satisfied and the lemma 1 holds. It follows that the Dirac operator $\mathcal{D}_{M}\left(x, \partial_{x}\right)$ can be obtained by projection of the operator $B^{a} \eta_{a}+B$ where $B^{a}$ and $B$ are given by (54), and we come to (53).

From this statement, we immediately obtain

Corollary 1. The generators

$$
\widetilde{X}(x)=\xi_{X}^{a}(x) \partial_{x^{a}}+\xi_{X}^{\alpha}\left(x, e_{H}\right) \Lambda_{\alpha}
$$

of representation of the Lie algebra $\mathfrak{g}$ in the space $V_{\Psi}$ are symmetry operators of the Dirac operator $\mathcal{D}_{M}$ on the homogeneous space $M$, i.e.,

$$
\begin{gathered}
{\left[\widetilde{X}(x), \mathcal{D}_{M}\left(x, \partial_{x}\right)\right]=0,} \\
{[\widetilde{X}(x), \widetilde{X}(x)]=\widetilde{[X, Y]}(x), \quad X, Y \in \mathfrak{g} .}
\end{gathered}
$$

In view of the isomorphism $\hat{\mathcal{F}} \simeq C^{\infty}\left(M, V_{\Psi}\right)$ and Theorem 1, the Dirac Equation (39) on $M$ is equivalent to the following system of equations on the transformation group $G$ :

$$
\left\{\begin{array}{l}
\mathcal{D}_{G}\left(g, \partial_{g}\right) \psi(g)=m \psi(g), \\
\left(\eta_{\alpha}+\Lambda_{\alpha}\right) \psi(g)=0
\end{array}\right.
$$

The function $\varphi(x)=\psi(s(x))=\psi\left(x, e_{H}\right)$ provides a solution of the original Dirac Equation (39) on the homogeneous space $M$.

\section{Non-Commutative Integration}

We will look for a solution to the system (61) as a set of functions

$$
\psi_{\sigma}(g)=\int_{Q} \psi_{\sigma}\left(q^{\prime}\right) D_{q q^{\prime}}^{\lambda}\left(g^{-1}\right) d \mu\left(q^{\prime}\right), \quad \sigma=(q, \lambda),
$$


where the function $\psi_{\sigma}\left(q^{\prime}\right)$ is a spinor, each component of which belongs to the function space $L(Q, \mathfrak{h}, \lambda)$ with respect to the variable $q^{\prime}$, and $D_{q q^{\prime}}^{\lambda}\left(g^{-1}\right)$ is introduced by (30).

Using (31), we can then reduce the system (61) to the equations

$$
\left\{\begin{array}{l}
\mathcal{D}_{\ell}\left(q^{\prime}, \partial_{q^{\prime}}, \lambda\right) \psi_{\sigma}\left(q^{\prime}\right)=m \psi_{\sigma}\left(q^{\prime}\right), \\
\left(\ell_{\alpha}\left(q^{\prime}, \lambda\right)+\Lambda_{\alpha}\right) \psi_{\sigma}\left(q^{\prime}\right)=0,
\end{array}\right.
$$

where

$$
\mathcal{D}_{\ell}\left(q^{\prime}, \partial_{q^{\prime}}, \lambda\right)=i \hbar \hat{\gamma}^{a}\left[\ell_{a}\left(q^{\prime}, \lambda\right)+\Gamma_{a}\right] .
$$

We call the operator $\mathcal{D}_{\ell}\left(q^{\prime}, \partial_{q^{\prime}}, \lambda\right)$ in (64) the Dirac operator in the $\lambda$-representation. The number of independent variables $q^{\prime}$ in (63) is $\operatorname{dim} Q$. The set of functions $\psi_{\sigma}(x)=\psi_{\sigma}(s(x))$ gives us a solution of the original Dirac equation (39) on the homogeneous space $M$.

It follows from the equations

$$
\xi_{X}(g) \psi_{\sigma}(g)=U(h) \widetilde{X}(x) \psi_{\sigma}(x)=-\ell_{X}(q, \lambda) \psi_{\sigma}(g)=U(h)\left\{-\ell_{X}(q, \lambda) \psi_{\sigma}(x)\right\}
$$

that the solutions $\psi_{\sigma}(x)$ of the Dirac equation satisfy the system

$$
\left\{\tilde{X}(x)+\ell_{X}(q, \lambda)\right\} \psi_{\sigma}(x)=0,
$$

where $\widetilde{X}$ is given by (14). The algebraic relations between operators of the $\lambda$-representation should correspond to the algebraic relations between the generators $\widetilde{X}(x)$ for compatibility of the system (65). More precisely, the corollary of the system (65) is to be fulfilled for any homogeneous function $F$ of $\widetilde{X}(x)$ :

$$
F(\widetilde{X}(x)) \psi_{\sigma}(x)=F\left(-\ell_{X}\left(q, \partial_{q}, \lambda\right)\right) \psi_{\sigma}(x) .
$$

This condition is obviously satisfied for the commutator of two operators $(X, Y \in \mathfrak{g})$,

$$
[\widetilde{X}(x), \widetilde{Y}(x)] \psi_{\sigma}(x)=-\left[\ell_{X}\left(q, \partial_{q}, \lambda\right), \ell_{Y}\left(q, \partial_{q}, \lambda\right)\right] \psi_{\sigma}(x),
$$

and for the Casimir functions, we have

$$
\begin{aligned}
& K_{\mu}^{(s)}(i \hbar \widetilde{X}) \psi_{\sigma}(x)=\left(U^{-1}(h) K_{\mu}^{(s)}(i \hbar \xi(g)) U(h)\right) \psi_{\sigma}(x)= \\
& =U^{-1}(h) K_{\mu}^{(s)}(i \hbar \xi(g)) \psi_{\sigma}(g)=\kappa_{\mu}^{(s)}(\lambda) \psi_{\sigma}(x) .
\end{aligned}
$$

The homogeneous functions $\Gamma \in C^{\infty}\left(\mathfrak{g}^{*}\right)$ provided that $\Gamma(X(x)) \equiv 0$ can exist on the dual space $\mathfrak{g}^{*}$ to the space $M$. These functions are called identities on the homogeneous space $M$. The number of functionally independent identities $i_{M}$ is called the index of the homogeneous space $M$. In Ref. [40], it was shown that any homogeneous function $\Gamma \in C^{\infty}\left(\mathfrak{g}^{*}\right)$ satisfying the condition

$$
\left.\Gamma(f)\right|_{f \in \mathfrak{h}^{\perp}}=0
$$

is an identity. In the same Ref. [40], it was shown that the functions $F_{\alpha}^{\left(s_{M}\right)}(f)$, where $s_{M}=$ $(\operatorname{dim} \mathfrak{g}-$ ind $\mathfrak{g}) / 2$, are identities, and all other identities are Casimir functions $K_{\mu}^{\left(s_{M}\right)}(f)$ such that

$$
\left.K_{\mu}^{\left(s_{M}\right)}(f)\right|_{f \in \mathfrak{h}^{\perp}} \equiv 0
$$

A description of identities for the generators $\widetilde{X}(x)$ of the form (14) is given by the following Lemma which is an essential result of our work: 
Lemma 3. The identities for the operators $\widetilde{X}(x)$ are generated by the functions $F_{\alpha}^{\left(s_{M}\right)}(f)$ and $K_{\mu}^{\left(s_{M}\right)}(f)$.

Proof. Suppose that a homogeneous function $\Gamma^{\prime} \in C^{\infty}\left(\mathfrak{g}^{*}\right)$ is an identity for the generators $\widetilde{X}(x)$, i.e., $\Gamma^{\prime}(\widetilde{X}) \equiv 0$. Then, the symbol

$$
\Gamma^{\prime}(x, p)=\Gamma^{\prime}(\widetilde{X}(x, p)) \in C^{\infty}\left(\mathfrak{g}^{*}, V\right)
$$

of the operator $\Gamma^{\prime}(\widetilde{X})$ also equals zero for all $(x, p)$, and

$$
\widetilde{X}(x, p)=X^{a}(x) p_{a}+\xi_{X}^{\alpha}\left(x, e_{H}\right) \Lambda_{\alpha}
$$

where the constants $p_{a}$ are coordinates of the covector $f^{x}=p_{a} d x^{a} \in T_{x}^{*} M$. At a given point $\left(x_{0}, p^{0}\right)$, we have

$$
\begin{gathered}
\widetilde{X}_{a}\left(x_{0}, p^{0}\right)=p_{a,}^{0} \quad \widetilde{X}_{\alpha}\left(x_{0}, p^{0}\right)=\Lambda_{\alpha}, \\
f^{x_{0}}=p_{a}^{0} d x^{0} \in T_{x_{0}}^{*} M \simeq \mathfrak{h}^{\perp} .
\end{gathered}
$$

Expanding $\Gamma^{\prime}(\widetilde{X}(x, p))$ in terms of the basis $B$ of matrices in the vector space $V$ and putting $x=x_{0}$, $p=f^{x_{0}}$, we get:

$$
\Gamma^{\prime}\left(\widetilde{X}\left(x_{0}, p^{0}\right)\right)=\Gamma^{\prime}\left(p_{1}^{0}, \ldots p_{\operatorname{dim} M}^{0}, \Lambda_{1}, \ldots, \Lambda_{\operatorname{dim} H}\right)=B^{\sigma} \Gamma_{\sigma}\left(p_{1}^{0}, \ldots p_{\operatorname{dim} M}^{0}\right) \equiv 0 .
$$

As a result, for each function $\Gamma_{\sigma}(f)$, we come to Equation (66). The last one shows that the functions $\Gamma_{\sigma}(f)$ are identities on a homogeneous space, and the identities $\Gamma^{\prime}(f)$ for the operators $\widetilde{X}(x)$ have the following structure:

$$
\begin{aligned}
\Gamma^{\prime}(\widetilde{X}(x)) & =\Gamma^{\prime}\left(X(x)+\xi^{\alpha}\left(x, e_{H}\right) \Lambda_{\alpha}\right)= \\
& =B^{\sigma} \Gamma_{\sigma}(X(x)) \equiv 0 .
\end{aligned}
$$

From this, one can see that the number of functionally independent identities between $\widetilde{X}(x)$ does not exceed the index $i_{M}$ of the homogeneous space, and the functions $\Gamma_{\sigma}(f)$ depend on identities on the homogeneous space.

For the compatibility of the system (65), we have to take into account the identities between the generators $\widetilde{X}(x), \Gamma^{\prime}(\widetilde{X}) \equiv 0$; namely, we impose the following conditions on the operators of the $\lambda$-representation:

$$
\Gamma^{\prime}\left(-\ell\left(q, \partial_{q}, \lambda\right)\right) \equiv 0
$$

A class of orbits and corresponding parameters $j$ should be restricted by (67).

For instance, for the case $\widetilde{X}(x)=X(x)$, the condition (67) is reduced to

$$
\begin{gathered}
F_{\alpha}^{\left(s_{M}\right)}\left(-i \hbar \ell\left(q, \partial_{q}, \lambda\right)\right) \equiv 0, \\
K_{\mu}^{\left(s_{M}\right)}\left(-i \hbar \ell\left(q, \partial_{q}, \lambda\right)\right)=\kappa_{\mu}^{\left(s_{M}\right)}(\lambda(j))=0 .
\end{gathered}
$$

The first condition in (68) says that the $\lambda$-representation has to be constructed by the class of orbits $\mathcal{O}_{\lambda(j)}^{\left(s_{M}\right)}$, and the second one imposes a restriction on the parameters $j$. In Ref. [44], a $\lambda$-representation satisfying (68) is called a $\lambda$-representation corresponding to the homogeneous space $M$.

Thus, condition (68) is stronger than (67). One of the important results of our work is the fact that, when performing a non-commutative reduction of the Dirac equation, it is necessary to use the weaker condition (67) for the correct application of the non-commutative integration method. 
The second equation of the system (63) can be written as

$$
\left(\alpha_{\alpha}^{\bar{a}}\left(q^{\prime}\right) \frac{\partial}{\partial q^{\prime \bar{a}}}+\frac{i}{\hbar} \xi_{\alpha}^{\bar{\alpha}}\left(q^{\prime}, e_{P}\right)\left(\lambda_{\bar{\alpha}}+i \hbar \beta_{\bar{\alpha}}\right)+\Lambda_{\alpha}\right) \psi_{\sigma}\left(q^{\prime}\right)=0 .
$$

We look for a solution of (69) in the form

$$
\psi_{\sigma}\left(q^{\prime}\right)=R_{\sigma}\left(q^{\prime}\right) \psi_{\sigma}(v)
$$

where $R_{\sigma}\left(q^{\prime}\right)$ is a certain function, and $\psi_{\sigma}(v)$ is an arbitrary function of the characteristics $v=v\left(q^{\prime}\right)$ of the system (63). We carry out a one-to-one change of variables $q^{\prime}=q^{\prime}(v, w)$, where $w=w\left(q^{\prime}\right)$ are some coordinates additional to $v$. By $V$ and $W$, we denote domains of the variables $v$ and $w$, respectively. The measure $d \mu\left(q^{\prime}\right)$ in the new variables takes the form $d \mu\left(q^{\prime}\right)=\rho(v, w) d \mu(v) d \mu(w)$. Then, the solution of the original Dirac equation can be represented as

$$
\begin{aligned}
& \psi_{\sigma}(x)=\int_{V} \psi_{\sigma}(v) D_{q v}^{\lambda}(x) d \mu(v) \\
& D_{q v}^{\lambda}(x)=\int_{W} R_{\sigma}(v, w) D_{q q^{\prime}(v, w)}^{\lambda}\left(g^{-1}\right) \rho(v, w) d \mu(w) .
\end{aligned}
$$

Substituting the solution $\psi_{\sigma}(x)$ into the Dirac equation (63), we obtain a linear first-order differential equation with matrix coefficients for the function $\psi_{\sigma}(v)$ with the number of independent variables $v$ equal to $\operatorname{dim} V$.

\section{The Metric That Does Not Admit Separation of Variables in the Dirac Equation}

Consider a four-dimensional homogeneous space $M$ with a transformation group $G$ whose Lie algebra $\mathfrak{g}$ is defined in some basis $\left\{e_{A}\right\}$ by nonzero commutation relations

$$
\begin{aligned}
& {\left[e_{1}, e_{4}\right]=-e_{1}, \quad\left[e_{1}, e_{5}\right]=e_{2}, \quad\left[e_{2}, e_{3}\right]=e_{1},} \\
& {\left[e_{2}, e_{4}\right]=e_{2}, \quad\left[e_{3}, e_{4}\right]=-2 e_{3}, \quad\left[e_{3}, e_{5}\right]=e_{4},} \\
& {\left[e_{4}, e_{5}\right]=-2 e_{5} .}
\end{aligned}
$$

The Lie algebra $\mathfrak{g}$ is a semidirect product of the two-dimensional commutative ideal $\mathbb{R}^{2}=\operatorname{span}\left\{e_{1}, e_{2}\right\}$ and the three-dimensional simple algebra $\mathfrak{s l}(2)=\operatorname{span}\left\{e_{3}, e_{4}, e_{5}\right\}$. We also take $\mathfrak{h}=\left\{e_{5}\right\}$ as the one-dimensional subalgebra.

Denote by $\left(x^{a}, h^{\alpha}\right)$ local coordinates on a trivialization domain $U$ of the group $G$ so that

$$
\begin{gathered}
g(x, h)=e^{h^{1} e_{5}} e^{x^{4} e_{4}} e^{x^{3} e_{3}} e^{x^{2} e_{2}} e^{x^{1} e_{1}} \\
x=\left(x^{1}, x^{2}, x^{3}, x^{4}\right) \in M .
\end{gathered}
$$

The group $G$ is unimodular and $\Delta_{G}(g)=1$. A symmetric non-degenerate matrix

$$
\left(G_{a b}\right)=\left(\begin{array}{cccc}
0 & 0 & 0 & -c_{3} \\
0 & c_{4} & c_{3} & c_{2} \\
0 & c_{3} & 0 & 0 \\
-c_{3} & c_{2} & 0 & c_{1}
\end{array}\right), \quad c_{k}=\text { const, } \quad c_{3} \neq 0,
$$

defines an invariant metric on the space $M$,

$$
\begin{aligned}
d s^{2} & =\frac{e^{x^{4}}}{c_{3}^{2}}\left\{2 c_{2} e^{2 x^{4}}\left(d x^{1}-x^{3} d x^{2}\right) d x^{3}-c_{1} e^{x^{4}}\left(d x^{1}-x^{3} d x^{2}\right)^{2}-c_{4} e^{3\left(x^{4}\right)^{2}} d\left(x^{3}\right)^{2}+\right. \\
& \left.+2 c_{3}\left(d x^{3}+x^{3} d x^{4}\right) d x^{2}-2 c_{3} d x^{1} d x^{4}\right\} .
\end{aligned}
$$


The metric (71) has nonzero scalar curvature $R=6 c_{1}$. The group generators in the canonical coordinates (70) have the form:

$$
\begin{aligned}
& X_{1}(x)=\partial_{x^{1}}, \quad X_{2}(x)=\partial_{x^{2}}, \quad X_{3}(x)=x^{2} \partial_{x^{1}}+\partial_{x^{3}}, \\
& X_{4}(x)=-x^{1} \partial_{x^{1}}+x^{2} \partial_{x^{2}}-2 x^{3} \partial_{x^{3}}+\partial_{x^{4}}, \\
& X_{5}(x)=x^{1} \partial_{x^{2}}-\left(x^{3}\right)^{2} \partial_{x^{3}}+x^{3} \partial_{x^{4}} .
\end{aligned}
$$

The vector fields $\xi_{A}\left(x, e_{H}\right)$, in turn, are determined by the expressions

$$
\xi_{a}(x, h)=X_{a}(x), \quad \xi_{5}(x, h)=X_{5}(x)+e^{-2 x^{4}} \partial_{h^{1}} .
$$

The right-invariant vector fields in the canonical coordinates (70) are

$$
\begin{gathered}
\eta_{1}(x, h)=-\left(e^{-x^{4}}+h_{1} x^{3} e^{x^{4}}\right) \partial_{x^{1}}-h_{1} e^{x^{4}} \partial_{x^{2}}, \quad \eta_{2}(x, h)=e^{-x^{4}}\left(x^{3} \partial_{x^{1}}+\partial_{x^{2}}\right), \\
\eta_{3}(x, h)=-e^{-2 x^{4}} \partial_{x^{3}}-h_{1} \partial_{x^{4}}+h_{1}^{2} \partial_{h^{1}}, \quad \eta_{4}(x, h)=-\partial_{x^{4}}+2 h_{1} \partial_{h^{1}}, \\
\eta_{5}\left(x, e_{H}\right)=-\partial_{h^{1}} .
\end{gathered}
$$

The gamma matrices $\hat{\gamma}^{a}$ can be presented in terms of the standard Dirac gamma matrices $\gamma^{a}$ as follows:

$$
\begin{aligned}
& \hat{\gamma}^{1}=\gamma^{1}+\gamma^{2}, \quad \hat{\gamma}^{2}=-\frac{c_{2}}{c_{3}}\left(\gamma^{1}+\gamma^{2}\right)+\sqrt{-c_{4}} \gamma^{3}, \\
& \hat{\gamma}^{3}=-\frac{c_{3}}{\sqrt{-c_{4}}}\left(\gamma^{3}+i \gamma^{4}\right), \\
& \hat{\gamma}^{4}=-\frac{c_{3}}{2}\left(\gamma^{1}-\gamma^{2}\right)-\frac{2 c_{2}}{\sqrt{-c_{4}}}\left(\gamma^{3}+i \gamma^{4}\right)-\frac{c_{1}}{c_{3}}\left(\gamma^{1}+\gamma^{2}\right) .
\end{aligned}
$$

The spin connection is independent of local coordinates and has the form

$$
\begin{aligned}
& \Gamma(x)=\Gamma-\hat{\gamma}^{4} \Lambda, \quad \Lambda=\frac{1}{2 c_{3}} \hat{\gamma}^{1} \hat{\gamma}^{3}, \\
& \Gamma=\frac{c_{1}}{4 c_{3}^{2}} \hat{\gamma}^{1} \hat{\gamma}^{1} \hat{\gamma}^{3}+\frac{c_{2}}{4 c_{3}^{2}} \hat{\gamma}^{1} \hat{\gamma}^{3} \hat{\gamma}^{4}+\frac{1}{c_{3}} \hat{\gamma}^{2} \hat{\gamma}^{3} \hat{\gamma}^{4}-\frac{c_{1}}{4 c_{3}} \hat{\gamma}^{1}+\frac{3 c_{3}}{4 c_{3}} \hat{\gamma}^{3}-2 \hat{\gamma}^{4} .
\end{aligned}
$$

The Dirac operator in local coordinates is

$$
\mathcal{D}_{M}=-i \hbar\left(\left[\hat{\gamma}^{1}+x^{3} e^{2 x^{4}} \hat{\gamma}^{2}\right] \partial_{y^{1}}+e^{x^{4}} \hat{\gamma}^{2} \partial_{x^{2}}-e^{-2 x^{4}} \hat{\gamma}^{3} \partial_{x^{3}}+\hat{\gamma}^{4} \partial_{x^{4}}\right)+\Gamma-\hat{\gamma}^{4} \Lambda .
$$

The first-order symmetry operators are defined by (60):

$$
\widetilde{X}_{a}(x)=X_{a}(x), \quad \widetilde{X}_{5}(x)=X_{5}(x)+e^{-2 x^{4}} \Lambda .
$$

The metric (71) generally does not admit the Yano vector field and the Yano-Killing tensor field, so the Dirac equation does not admit spin symmetry operators. As a result, the Dirac equation has only two commuting symmetry operators $\left\{\widetilde{X}_{1}(x), \widetilde{X}_{2}(x)\right\}$ of the first order. However, the Dirac equation admits a third-order symmetry operator

$$
K(\widetilde{X})=\widetilde{X}_{5}(x) \cdot \widetilde{X}_{1}(x) \cdot \widetilde{X}_{1}(x)+\widetilde{X}_{1}(x) \cdot \widetilde{X}_{2}(x) \cdot \widetilde{X}_{4}(x)-\widetilde{X}_{2}(x) \cdot \widetilde{X}_{2}(x) \cdot \widetilde{X}_{4}(x),
$$

where $X \cdot Y=(X Y+Y X) / 2$ is the symmetrized product of the operators $X$ and $Y$. As a consequence, the metric (71) does not admit separation of variables for the Dirac equation. Note that the Klein-Gordon equation also admits only three commuting symmetry operators $\left\{X_{1}(x), X_{2}(x), K(X)\right\}$. 
One of them, $K(X)$, is the third-order operator, and the Klein-Gordon equation also does not admit separation of variables.

We now carry out a non-commutative reduction of the Dirac equation.

First, we describe orbits of the coadjoint representation of the Lie group $G$. The Lie algebra $\mathfrak{g}$ admits the Casimir function $K(f)=f_{5} f_{1}^{2}+f_{2} f_{4} f_{1}-f_{2}^{2} f_{3}$. Expansion (23) takes the form

$$
\begin{aligned}
& M_{0}=\left\{f \in \mathfrak{g}^{*} \mid \neg\left(f_{1}=f_{2}=0\right)\right\}, \quad \mathcal{O}_{\omega}^{0}=\left\{f \in M_{0} \mid K_{1}(f)=\omega_{1}^{0}\right\}, \\
& \Omega^{0}=\mathbb{R}^{1}, \quad \operatorname{dim} \mathcal{O}_{\lambda}^{0}=4, \\
& M_{1}=\left\{f \in \mathfrak{g}^{*} \mid f_{1}=f_{2}=0, \neg(f=0)\right\}, \quad \mathcal{O}_{\omega^{1}}^{1}=\left\{f \in M_{0} \mid K_{1}^{(1)}(f)=\omega_{1}^{1}\right\}, \\
& M_{2}=\mathcal{O}^{2}=\{f=0\}, \\
& \mathfrak{g}^{*}=M_{0} \cup M_{1} \cup M_{2}, \quad M_{0}=\bigcup_{\omega^{0} \in \Omega^{0}} \mathcal{O}_{\omega}^{0}, \quad M_{1}=\bigcup_{\omega^{1} \in \Omega^{1}} \mathcal{O}_{\omega^{1}}^{1},
\end{aligned}
$$

where $K_{1}^{(1)}(f)=f_{4}^{2}+4 f_{3} f_{5}$ is the Casimir function of $s=1$ type. Each non-degenerate orbit from the class $\mathcal{O}_{\omega}^{0}$ passes through the covector $\lambda(j)=(1,0,0,0, j)$ and $K_{1}(\lambda(j))=j$, where $j \in \mathbb{R}$.

The covector $\lambda(j)$ admits a real polarization $\mathfrak{p}=\left\{e_{1}, e_{2}, e_{5}\right\}$, and the $\lambda$-representation corresponding to the class of orbits $\mathcal{O}_{\lambda(j)}^{0}$ is given by

$$
\begin{aligned}
& \ell_{1}\left(q, \partial_{q}, \lambda\right)=\frac{i}{\hbar} q_{1}, \quad \ell_{2}\left(q, \partial_{q}, \lambda\right)=-\frac{i}{\hbar} q_{2}, \\
& \ell_{3}\left(q, \partial_{q}, \lambda\right)=q_{1} \partial_{q^{2}}, \quad \ell_{4}\left(q, \partial_{q}, \lambda\right)=q_{1} \partial_{q^{1}}-q_{2} \partial_{q^{2}}, \\
& \ell_{5}\left(q, \partial_{q}, \lambda\right)=q_{2} \partial_{q^{1}}+\frac{i}{\hbar} \frac{j}{q_{1}^{2}} \\
& K_{1}(-i \hbar \ell)=j .
\end{aligned}
$$

The operators $-i \hbar \ell_{X}\left(q, \partial_{q}, \lambda\right)$ are symmetric with respect to the measure $d \mu(q)=d q_{1} d q_{2}, Q=\mathbb{R}^{2}$. Now solving Equation (31), we get

$$
\begin{aligned}
D_{q q^{\prime}}^{\lambda}\left(g^{-1}\right)= & \exp \left(\frac{i}{\hbar}\left[q_{2} x^{2}-q_{1} x^{1}+\frac{j}{q_{2}^{\prime}}\left(\frac{e^{x^{4}}}{q_{1}}-\frac{1}{q_{1}^{\prime}}\right)\right]\right) \times \\
& \times \delta\left(-h_{1} q_{2}^{\prime}+q_{1} e^{-x^{4}}, q_{1}^{\prime}\right) \delta\left(-e^{x^{4}}\left(q_{1} x^{3}-q_{2}\right), q_{2}^{\prime}\right),
\end{aligned}
$$

where $\delta\left(q, q^{\prime}\right)$ is the generalized Dirac delta function. The completeness and orthogonality conditions (36) and (35) are satisfied for the measure

$$
d \mu(\lambda)=(2 \pi \hbar)^{-3} d j, \quad j \in \mathbb{R}^{1} .
$$

Orbits from the class $\mathcal{O}_{\lambda(j)}^{0}$ satisfy the integral condition (34).

The homogeneous space $M$ has zero index, $i_{M}=0$, and does not have identities that have to be taken into account in the method of non-commutative integration. Thus, the $\lambda$-representation (73) corresponds to the homogeneous space $M$.

Integrating the equation

$$
\left(\ell_{5}\left(q^{\prime}, \partial_{q^{\prime}}, \lambda\right)+\Lambda\right) \psi_{\sigma}\left(q^{\prime}\right)=0, \quad \sigma=\left(q_{1}, q_{2}, j\right)
$$

yields

$$
\psi_{\sigma}\left(q^{\prime}\right)=\exp \left(\frac{i j}{\hbar q_{1}^{\prime} q_{2}^{\prime}}-\frac{q_{1}^{\prime}}{q_{2}^{\prime}} \Lambda\right) \psi_{\sigma}\left(q_{2}^{\prime}\right)
$$


Substituting (74) into the Dirac equation in the $\lambda$-representation (63), we obtain the ordinary differential equation for the spinor $\psi_{\sigma}$,

$$
\begin{aligned}
& i \hbar \frac{d}{d q_{2}^{\prime}} \psi_{\sigma}\left(q_{2}^{\prime}\right)=M\left(q_{2}^{\prime}\right) \psi_{\sigma}\left(q_{2}^{\prime}\right), \\
& M\left(q_{2}^{\prime}\right)=\frac{1}{c_{1} q_{2}^{\prime}} \hat{\gamma}^{4}\left\{i \hbar \Gamma+q_{2}^{\prime} \hat{\gamma}^{2}+\frac{j}{q_{2}^{\prime 2}} \hat{\gamma}^{3}-m\right\} .
\end{aligned}
$$

Then, we obtain the solution as

$$
\psi_{\sigma}\left(q_{2}^{\prime}\right)=C_{\sigma} \exp \left(-\frac{i}{\hbar} \int_{1}^{q_{2}^{\prime}} M(u) d u\right)
$$

where $C_{\sigma}$ is the normalization factor.

The solution to the Dirac equation in local coordinates can be obtained by substituting (74) into (62) and integrating over $q^{\prime}$ for $h_{1}=0$ :

$$
\psi_{\sigma}(x)=\exp \left(\frac{\frac{i j}{\hbar q_{1}}-q_{1} e^{-2 x^{4}} \Lambda}{q_{2}-q_{1} x^{3}}\right) \exp \left(\frac{i}{\hbar}\left[q_{2} x^{2}-q_{1} x^{1}\right]\right) \psi_{\sigma}\left(e^{x^{4}}\left(q_{2}-q_{1} x^{3}\right)\right) .
$$

Equations (75) and (76) provide a complete and orthogonal set of solutions to the Dirac equation on the homogeneous space $M$ with the metric (71).

\section{The Dirac Equation in $\operatorname{AdS}_{3}$ Space}

Consider a three-dimensional de Sitter space $M$ as a homogeneous space with the de Sitter group of transformations $G=S O(1,3)$ and the isotropy subgroup $H$ being the Lorentz group $S O(1,2)$. The de Sitter space $M$ has constant positive curvature and $M$ is topologically isomorphic to $R^{1} \times S^{2}$.

The group $S O(1,3)$ is defined as a rotation group of a four-dimensional pseudo-Euclidean space with the metric $\left(G_{A B}\right)=\operatorname{diag}(1,-1,-1,-1)$. The Lie algebra $\mathfrak{g}=\mathfrak{s o}(1,3)$ of the group $S O(1,3)$ in the basis $\left\{E_{A B} \mid A<B\right\}$ is defined by the commutation relations

$$
\left[E_{A B}, E_{C D}\right]=G_{A D} E_{B C}-G_{A C} E_{B D}+G_{B C} E_{A D}-G_{B D} E_{A C},
$$

where $A, B, C, D=1, \ldots, 4$. The basis $E_{A B}$ can be represented as

$$
\begin{gathered}
E_{a b}=e_{a b},(a<b), \quad E_{a 4}=e_{a} / \varepsilon, \\
{\left[e_{a}, e_{b}\right]=\varepsilon^{2} e_{a b}, \quad a, b=1, \ldots, 3 .}
\end{gathered}
$$

Here, the basis $e_{a b}$ forms the isotropy subalgebra $\mathfrak{h}=\mathfrak{s o}(1,2)$, and $\varepsilon$ is a parameter defining the curvature of the de Sitter space. We introduce the canonical coordinates of the second kind on the Lie group $G$ so that

$$
g\left(t, x, y, h_{1}, h_{2}, h_{3}\right)=e^{h_{3} e_{23}} e^{h_{2} e_{13}} e^{h_{1} e_{12}} e^{y e_{3}} e^{x e_{2}} e^{t e_{1}} .
$$

The group generators in the canonical coordinates can be written as 


$$
\begin{aligned}
X_{1}(x) & =\partial_{t} \\
X_{2}(x) & =\sinh (\varepsilon t) \tan (\varepsilon x) \partial_{t}+\cosh (\varepsilon t) \partial_{x}, \\
X_{3}(x) & =\sinh (\varepsilon t) \sec (\varepsilon x) \tan (\varepsilon y) \partial_{t}+ \\
& \cosh (\varepsilon t) \sin (\varepsilon x) \tan (\varepsilon y) \partial_{x}+ \\
& \cosh (\varepsilon t) \cos (\varepsilon x) \partial_{y}, \\
X_{12}(x) & =\varepsilon^{-1}\left(\cosh (\varepsilon t) \tan (\varepsilon x) \partial_{t}+\sinh (\varepsilon t) \partial_{x}\right), \\
X_{13}(x) & =\varepsilon^{-1}\left(\cosh (\varepsilon t) \sec (\varepsilon x) \tan (\varepsilon y) \partial_{t}+\right. \\
& \sinh (\varepsilon t) \sin (\varepsilon x) \tan (\varepsilon y) \partial_{x}+ \\
& \left.\sinh (\varepsilon t) \cos (\varepsilon x) \partial_{y}\right), \\
X_{23}(x) & =\varepsilon^{-1}\left(\cos (\varepsilon x) \tan (\varepsilon y) \partial_{x}-\sin (\varepsilon x) \partial_{y}\right) .
\end{aligned}
$$

The vector fields $\xi\left(x, e_{H}\right)$, in turn, are determined by

$$
\begin{aligned}
& \xi_{1}\left(x, e_{H}\right)=X_{1}(x) \\
& \xi_{2}\left(x, e_{H}\right)=X_{2}(x)+\epsilon \sinh (t \epsilon) \sec (x \epsilon) \partial_{h^{1}} \\
& \xi_{3}\left(x, e_{H}\right)=X_{3}(x)+\epsilon \sinh (t \epsilon) \tan (x \epsilon) \tan (y \epsilon) \partial_{h^{1}} \\
& +\epsilon \sinh (t \epsilon) \sec (y \epsilon) \partial_{h^{2}}+ \\
& \epsilon \cosh (t \epsilon) \sin (x \epsilon) \sec (y \epsilon) \partial_{h^{3}} \\
& \xi_{12}\left(x, e_{H}\right)=X_{12}(x)+\cosh (t \epsilon) \sec (x \epsilon) \partial_{h^{1}} \\
& \xi_{13}\left(x, e_{H}\right)=X_{13}(x)+\cosh (t \epsilon) \tan (x \epsilon) \tan (y \epsilon) \partial_{h^{1}} \\
& +\cosh (t \epsilon) \sec (y \epsilon) \partial_{h^{2}}+ \\
& \sinh (t \epsilon) \sin (x \epsilon) \sec (y \epsilon) \partial_{h^{3}} \\
& \xi_{14}\left(x, e_{H}\right)=X_{14}(x)+\cos (x \epsilon) \sec (y \epsilon) \partial_{h^{3}} .
\end{aligned}
$$

The right-invariant vector fields in the canonical coordinates (77) are

$$
\begin{aligned}
\eta_{1}\left(x, e_{H}\right)= & -\sec (\varepsilon x) \sec (\varepsilon y) \partial_{x}- \\
& \varepsilon \tan (\varepsilon x) \sec (\varepsilon y) \partial_{h^{1}}-\varepsilon \tan (\varepsilon y) \partial_{h^{2}}, \\
\eta_{2}\left(x, e_{H}\right)= & -\sec (\varepsilon y) \partial_{y}-\varepsilon \tan (\varepsilon y) \partial_{h^{3}} \\
\eta_{3}\left(x, e_{H}\right)= & -\partial_{z}, \quad \eta_{12}\left(x, e_{H}\right)=-\partial_{h^{1}} \\
\eta_{13}\left(x, e_{H}\right)= & -\partial_{h^{2}}, \quad \eta_{23}\left(x, e_{H}\right)=-\partial_{h^{3}} .
\end{aligned}
$$

The 2-form $\left(G_{a b}\right)=\operatorname{diag}(1,-1,-1)$ defines an invariant metric on the space $M$ in local coordinates as

$$
\begin{aligned}
& d s^{2}=g_{i j}(x) d x^{i} d x^{j}=\rho(x, y)^{2}\left\{d t^{2}-\frac{d x^{2}}{\cos ^{2}(\varepsilon x)}-\frac{d y^{2}}{\rho(x, y)^{2}}\right\}, \\
& \rho(x, y)=\cos (\varepsilon x) \cos (\varepsilon y),
\end{aligned}
$$

whence the scalar curvature of the space $M$ reads $R=6 \varepsilon^{2}$.

The gamma matrices $\hat{\gamma}^{a}$ in the $(2+1)$-dimensional space in terms of the Pauli matrices $\left\{\sigma_{x}, \sigma_{y}, \sigma_{z}\right\}$ are

$$
\hat{\gamma}^{1}=\sigma_{z}, \quad \hat{\gamma}^{2}=i s \sigma_{x}, \quad \hat{\gamma}^{3}=i \sigma_{y} .
$$


Here, the parameter $s=\operatorname{Tr} \hat{\gamma}^{1} \hat{\gamma}^{2} \hat{\gamma}^{3}= \pm 1$ is called a pseudospin. The matrices

$$
\Lambda_{12}=-\frac{i s}{2} \hat{\gamma}^{3}, \quad \Lambda_{13}=\frac{i s}{2} \hat{\gamma}^{2}, \quad \Lambda_{23}=-\frac{i s}{2} \hat{\gamma}^{1}
$$

realize a spinor representation of the isotropy subalgebra in a space of two-dimensional spinors. In our case $\Gamma_{a}=0$ and the spin connection takes the simple form

$$
\Gamma(x)=\hat{\gamma}^{a} \eta_{a}^{\alpha}\left(x, e_{H}\right) \Lambda_{\alpha}=\frac{\varepsilon}{2} \hat{\gamma}^{2} \tan (\varepsilon x) \sec (\varepsilon y)+\varepsilon \hat{\gamma}^{3} \tan (\varepsilon y) .
$$

Then, the Dirac operator in local coordinates reads

$$
\mathcal{D}_{M}=-i \sec (\varepsilon x) \sec (\varepsilon y) \hat{\gamma}^{1} \partial_{t}-i \sec (\varepsilon y) \hat{\gamma}^{2}\left(\partial_{x}-\frac{\varepsilon}{2} \tan (\varepsilon x)\right)-i \hat{\gamma}^{3}\left(\partial_{y}-\varepsilon \tan (\varepsilon y)\right) .
$$

The first-order symmetry operators, as defined by (60), are given by

$$
\begin{aligned}
\widetilde{X}_{1}(x) & =X_{1}(x) \\
\widetilde{X}_{2}(x) & =X_{2}(x)-\frac{i s \varepsilon}{2} \sinh (\varepsilon t) \sec (\varepsilon x) \hat{\gamma}^{3} \\
\widetilde{X}_{3}(x) & =X_{3}(x)-\frac{i s \varepsilon}{2}\left(\cosh (\varepsilon t) \sin (\varepsilon x) \sec (\varepsilon y) \hat{\gamma}^{1}-\right. \\
& \sinh (\varepsilon t) \sec (\varepsilon y) \hat{\gamma}^{2}- \\
& \left.\sinh (\varepsilon t) \tan (\varepsilon x) \tan (\varepsilon y) \hat{\gamma}^{3}\right) \\
\widetilde{X}_{12}(x) & =X_{12}(x)-\frac{i s \varepsilon}{2} \cosh (\varepsilon t) \sec (\varepsilon x) \hat{\gamma}^{3} \\
\widetilde{X}_{13}(x) & =X_{13}(x)-\frac{i s}{2}\left(\sinh (\varepsilon t) \sin (\varepsilon x) \sec (\varepsilon y) \hat{\gamma}^{1}-\right. \\
& \cosh (\varepsilon t) \sec (\varepsilon y) \hat{\gamma}^{2}+ \\
& \left.\cosh (\varepsilon t) \tan (\varepsilon x) \tan (\varepsilon y) \hat{\gamma}^{3}\right) \\
\widetilde{X}_{14}(x) & =X_{14}(x)-\frac{i s \varepsilon}{2} \cos (\varepsilon x) \sec (\varepsilon y) \hat{\gamma}^{1} .
\end{aligned}
$$

Our aim is to construct a complete set of exact solutions of the Dirac equation corresponding to the operator $\mathcal{D}_{M}$ using the non-commutative integration method.

The Lie algebra $\mathfrak{g}$ admits the following two Casimir functions:

$$
K_{1}(f)=p^{a} p_{a}-\frac{\varepsilon^{2}}{2} l^{a b} l_{a b}, \quad K_{2}(f)=\frac{\varepsilon}{8} e^{A B C D} L_{A B} L_{C D},
$$

where $e^{A B C D}$ is the Levi-Civita symbol $\left(e^{1234}=1\right) ;\left(p_{a}, l_{a b}\right)$ are coordinates of the covector with respect to the basis $\left\{e^{a}, e^{a b}\right\}$, i.e., $f=p_{a} e^{a}+l_{a b} e^{a b}$, and $L_{A B}$ are the coordinates with respect to the basis $\left\{E^{A B}\right\}, f=L_{A B} E^{A B}$. The raising and lowering indices are performed using the matrix $G_{A B}$. The expansion (23) in our case takes the form:

$$
\begin{aligned}
& M_{0}=\left\{f \in \mathfrak{g}^{*} \mid \neg(f=0)\right\}, \\
& \mathcal{O}_{\omega}^{0}=\left\{f \in M_{0} \mid K_{1}(f)=\omega_{1}^{0}, \quad K_{2}(f)=\omega_{2}^{0}\right\}, \\
& \Omega^{0}=\mathbb{R}^{2}, \quad \operatorname{dim} \mathcal{O}_{\lambda}^{0}=4, \\
& M_{1}=\{\varnothing\}, \quad \mathcal{O}^{1}=\{\varnothing\}, \quad M_{2}=\mathcal{O}^{2}=\{f=0\}, \\
& \mathfrak{g}^{*}=M_{0} \cup M_{2}, \quad M_{0}=\bigcup_{\omega^{0} \in \Omega^{0}} \mathcal{O}_{\omega}^{0} .
\end{aligned}
$$


Each non-degenerate orbit from the class $\mathcal{O}_{\omega}^{0}$ passes through the covector $\lambda(j)=\left(j_{1}, 0,0,0,0, j_{2}\right)$ characterized by two real parameters $j=\left(j_{1}, j_{2}\right) \in \mathbb{R}^{2}$, and

$$
\begin{aligned}
& K_{1}(\lambda(j))=\omega_{1}^{0}(j)=j_{1}^{2}-\varepsilon^{2} j_{2}^{2}, \quad K_{2}(\lambda(j))=\omega_{2}^{0}(j)=j_{1} j_{2}, \\
& \operatorname{det}\left\|\frac{\partial \omega_{\mu}^{0}(j)}{\partial j_{v}}\right\|=2\left(j_{1}^{2}+\varepsilon^{2} j_{2}^{2}\right) .
\end{aligned}
$$

If $K_{2}(f)=0$ for $f \in \mathfrak{p}^{\perp}=\left\{f_{a} e^{a} \mid f \in \mathfrak{g}^{*}\right\}$, then the Casimir operator $K_{2}(X)$ is an identity on the homogeneous space $M$ :

$$
K_{2}(X)=X_{3} \cdot X_{12}-X_{2} \cdot X_{13}+X_{1} \cdot X_{23}=0 .
$$

Note that $K_{2}(\widetilde{X})$ is proportional to the Dirac operator:

$$
K_{2}(\widetilde{X})=K_{2}(X)=\widetilde{X}_{3} \cdot \widetilde{X}_{12}-\widetilde{X}_{2} \cdot \widetilde{X}_{13}+\widetilde{X}_{1} \cdot \widetilde{X}_{23}=\frac{s}{2} \mathcal{D}_{M} .
$$

The covector $\lambda(j)$ admits the real polarization

$$
\mathfrak{p}=\left\{e_{1}, e_{2}+\varepsilon e_{12}, e_{3}+\varepsilon e_{13}, e_{23}\right\} .
$$

The corresponding $\lambda$-representation for the class of orbits $\mathcal{O}_{\lambda(j)}^{0}$ is represented in Appendix A (see Equation (A1)). The Casimir operators in the $\lambda$-representation are

$$
\begin{aligned}
& K_{1}(-i \hbar \ell)=\kappa_{2}(\lambda)=j_{1}^{2}-\varepsilon^{2} j_{2}^{2}+(\varepsilon \hbar)^{2}, \\
& K_{2}(-i \hbar \ell)=\kappa_{2}(\lambda)=j_{1} j_{2} .
\end{aligned}
$$

The equation $\left(\ell_{\alpha}\left(q^{\prime}, \partial_{q^{\prime}}, \lambda\right)+\Lambda_{\alpha}\right) c_{\lambda}\left(q^{\prime}\right)=0$ provided that $j_{2}=s / 2$ has a nonzero solution

$$
\begin{aligned}
c_{\lambda}(q) & =\frac{\left(\cos \left(\varepsilon q_{1}\right) \cos \left(\varepsilon q_{2}\right)\right)^{-\frac{3}{2}-\frac{i j_{1}}{\hbar \varepsilon}}}{\cos \left(\varepsilon q_{1}\right) \cos \left(\varepsilon q_{2}\right)+1} \times \\
& \times\left(\cos \left(\frac{\varepsilon\left(q_{1}+q_{2}\right)}{2}\right)+i s \cos \left(\frac{\varepsilon\left(q_{1}-q_{2}\right)}{2}\right)\right)\left(\begin{array}{c}
\cos \left(\varepsilon q_{1}\right) \cos \left(\varepsilon q_{2}\right)+1 \\
i s \sin \left(\varepsilon q_{1}\right)-\cos \left(\varepsilon q_{1}\right) \sin \left(\varepsilon q_{2}\right)
\end{array}\right) .
\end{aligned}
$$

The Dirac equation in the $\lambda$-representation,

$$
\left\{i \hbar \hat{\gamma}^{a} \ell_{a}\left(q^{\prime}, \partial_{q^{\prime}}, \lambda\right)-m\right\} c_{\lambda}\left(q^{\prime}\right)=0,
$$

is reduced to the algebraic equation $j_{1}+m=0$, then we have $j_{1}=-m$ and $j_{2}=s / 2$. That is, the eigenvalue of the Casimir operator $K_{1}(i \hbar X)$ is determined by the particle mass $m$, and the eigenvalue of the second Casimir operator, $K_{2}(i \hbar X)$, depends on the parameter $s$ :

$$
\kappa_{1}(\lambda)=m^{2}-\frac{1}{4} \varepsilon^{2}+(\varepsilon \hbar)^{2}, \quad \kappa_{2}(\lambda)=-\frac{1}{2} m s .
$$

The solution of the original Dirac equation in our case reads

$$
\psi_{\sigma}(x)=e^{-t \ell_{1}\left(q, \partial_{q}, \lambda\right)} e^{-x \ell_{2}\left(q, \partial_{q}, \lambda\right)} e^{-y \ell_{3}\left(q, \partial_{q} \cdot \lambda\right)} c_{\lambda}(q), \quad \sigma=\left(q_{1}, q_{2}\right) .
$$

Here, the exponentials of operators of the $\lambda$-representation for the fixed $j_{1}=-m$ and $j_{2}=s / 2$ act on a function according to Appendix A, Equation (A2). From here, one can see that the solution (78) depends on two quantum numbers $q_{1}$ and $q_{2}$, which are not eigenvalues for symmetry operators. The explicit form of the solution (78) is cumbersome, but it is expressed in terms of elementary functions. 


\section{Conclusions}

In this paper, we have explored the Dirac equation with an invariant metric on a homogeneous space $M$ of arbitrary dimension and developed the non-commutative integration method for this equation based on the ideas of symmetry analysis and the Lie group theory.

The Dirac equation and its symmetry are convenient to study in terms of algebraic structures associated with homogeneous spaces, and the theory of Lie group representations can be effectively applied for constructing exact solutions.

Using a special choice of the local frame and right-invariant vector fields on the Lie group of transformations $G$, we have obtained the spin connection (45). The Dirac equation is shown to be equivalent to a system of linear differential equations with constant matrix coefficients on the Lie group $G$ given by (61) that is the starting point for non-commutative integration.

In Refs. [22-27], an early version of the NCIM was used to construct exact solutions to the Dirac equation in four-dimensional space-time where the $\lambda$-representation was constructed directly by definition (28), and the domain of the variables $q$ was not associated with a Lagrangian submanifold to the K-orbit. The desired solutions were constructed by means of joint integration of the system (65) in local coordinates together with the original Dirac Equation (39).

Differently to the above early method, the main idea here is the non-commutative reduction of the corresponding system of equations on the Lie group $G$ and the connection between the solutions of this system and the original Dirac equation.

The non-commutative reduction is defined here using a special irreducible $\lambda$-representation of the Lie algebra $\mathfrak{g}$ of the Lie group $G$, which we introduce using the orbit method [37]. The key point of the method developed is based on the fact that there exist the identities connecting symmetry operators on a homogeneous space. For the Dirac equation, as follows from the lemma 3, the number of identities is either less than for the Klein-Gordon equation or they are completely absent. For the Klein-Gordon equation, the number of identities is determined by the index of the homogeneous space [19].

The problem of describing identities for the symmetry operators of the Dirac equation on the homogeneous space is constructively solved for the first time. The reduced system (63) in the $\lambda$-representation depending on a smaller number of independent variables $q^{\prime}$ is obtained. What is remarkable is the fact that the solutions obtained for the Dirac equation on a homogeneous space are closely related to the two principal bundles of the transformation group. The local coordinates $x$ on the space $M$ are determined by means of the principal $H$-bundle of the group $G$ with the isotropy subgroup $H$ of $M$, and the set of quantum numbers $q$ is connected with the principal $P$-bundle of the same group $G$ and the subgroup $P$.

The NCIM developed in the paper for the Dirac equation is illustrated by two non-trivial examples. In one of them, described in Section 7, we have found by using the NCIM a complete set of solutions to the Dirac equation (the MCIM-solutions) in the case when the metric does not admit separation of variables neither in the Klein-Gordon equation nor in the Dirac equation. The solutions obtained are eigenfunctions of the symmetry operator of the third-order (72) and are parameterized by three parameters $\left(q_{1}, q_{2}, j\right)$.

The second example is the three-dimensional de Sitter space $\mathrm{AdS}_{3}$ with the transformation group $S O(1,3)$ (Section 8). In this case, the Casimir operator $K_{2}(-i \hbar \widetilde{X})$ is proportional to the Dirac operator:

$$
\mathcal{D}_{M}=2 s K(-i \hbar \widetilde{X}),
$$

and the spectrum of the Casimir operator $-K_{1}(-i \hbar \widetilde{X})$ gives the mass $m$ of the spinor field.

It is worth noting that the function $K_{2}(f)$ is an identity in the homogeneous space $M$, but when substituting the extended operators $-i \hbar \widetilde{X}$, it is no longer an identity, and this leads to the original Dirac operator. If we consider the Klein-Gordon equation in $\mathrm{AdS}_{3}$, the operator $-K_{1}(-i \hbar \widetilde{X})$ is proportional to the operator of the equation, and the second operator $K_{2}(-i \hbar X)$ is identically zero that corresponds to the case $s=0$. Thus, the non-commutative integration of the Dirac equation is different from the 
non-commutative integration of the Klein-Gordon equation. The complete set of exact solutions (78) of the Dirac equation found by the NCIM is parameterized by two real parameters $\left(q_{1}, q_{2}\right)$ and is expressed by means of elementary functions, while the separation of variables gives the basis solutions to the Dirac equation in terms of special functions.

The parameters $q$ of solutions (76) and (78) obtained by the NCIM are in general not eigenvalues of an operator, a fact that crucially distinguishes them from solutions obtained by a separation of variables. Nevertheless, the NCIM-solutions can be effectively applied in order to study quantum effects in homogeneous spaces (see, e.g., [19,20]).

The NCIM-solutions of the Dirac equation may have a wide range of applications in the theory of fermion fields [45,46], quantum cosmology [47,48], and other problems of field theory. The NCIM can also be applied to the Dirac-type equation for theoretical models in the condensed matter (graphene, topological insulators, etc.) $[49,50]$. Note that the technique proposed in the article can be easily generalized to the case of spaces having new spatial dimensions much larger than the weak scale, as large as a millimeter for the case of two extra dimensions [51].

Finally, we note that the NCIM reveals new aspects, both related to the symmetry of the Dirac equation and its integrability, and to study the properties of new solutions constructed. One of the problems is to find out the meaning of the parameters $q$ entering into the NCIM-solutions which, in the general case, do not have to be eigenvalues of operators representing observables. One can notice some similarity of the NCIM-solutions with well-studied coherent states [52]. In particular, the action of the group on the set of $Q$ data of quantum numbers is defined that can be found in the theory of coherent states [52-55]. However, the analysis of the parameters is the subject of special research.

Author Contributions: Conceptualization, A.S. and A.B.; methodology, A.S.; formal analysis, A.S. and A.B.; investigation, A.S. and A.B.; validation, A.S. and A.B.; writing-original draft, A.S.; writing-review and editing, A.S. All authors have read and agreed to the published version of the manuscript.

Funding: This research was funded by RFBR, Grant No. 20-01-00389 A and Grant No. 19-41-700004.

Acknowledgments: Breev and Shapovalov were partially supported by Tomsk State University under the International Competitiveness Improvement Program; Shapovalov was partially supported by Tomsk Polytechnic University under the International Competitiveness Improvement Program.

Conflicts of Interest: The authors declare no conflict of interest.

\section{Appendix A. $\lambda$-Representation of Lie Algebra $\mathfrak{s o}(1,3)$}

The $\lambda$-representation for the class of orbits $\mathcal{O}_{\lambda(j)}^{0}$ is written as

$$
\begin{aligned}
\ell_{1}\left(q, \partial_{q}, \lambda\right)= & \sin \left(\varepsilon q_{1}\right) \cos \left(\varepsilon q_{2}\right) \partial_{q^{1}}+\sec \left(\varepsilon q_{1}\right) \sin \left(\varepsilon q_{2}\right) \partial_{q^{2}}+ \\
+ & \left(\varepsilon+\frac{i}{\hbar} j_{1}\right) \cos \left(\varepsilon q_{1}\right) \cos \left(\varepsilon q_{2}\right)+\frac{i}{\hbar} \varepsilon j_{2} \tan \left(\varepsilon q_{1}\right) \sin \left(\varepsilon q_{2}\right) \\
\ell_{2}\left(q, \partial_{q}, \lambda\right)= & \cos \left(\varepsilon q_{2}\right) \partial_{q^{1}}+\tan \left(\varepsilon q_{1}\right) \sin \left(\varepsilon q_{2}\right) \partial_{q^{2}}-\frac{i}{\hbar} \varepsilon j_{2} \sec \left(\varepsilon q_{1}\right) \sin \left(\varepsilon q_{2}\right), \\
\ell_{3}\left(q, \partial_{q}, \lambda\right)= & \partial_{q^{2}}, \\
\ell_{12}\left(q, \partial_{q}, \lambda\right)= & \varepsilon^{-1}\left[-\cos \left(\varepsilon q_{1}\right) \partial_{q^{1}}+\left(\varepsilon+\frac{i}{\hbar} j_{1}\right) \sin \left(\varepsilon q_{1}\right)\right] \\
\ell_{13}\left(q, \partial_{q}, \lambda\right)= & \varepsilon^{-1}\left[\sin \left(\varepsilon q_{1}\right) \sin \left(\varepsilon q_{2}\right) \partial_{q^{1}}-\sec \left(\varepsilon q_{1}\right) \cos \left(\varepsilon q_{2}\right) \partial_{q^{2}}+\right. \\
& \left.+\left(\varepsilon+\frac{i}{\hbar} j_{1}\right) \cos \left(\varepsilon q_{1}\right) \sin \left(\varepsilon q_{2}\right)+\frac{i}{\hbar} \varepsilon j_{2} \tan \left(\varepsilon q_{1}\right) \cos \left(\varepsilon q_{2}\right)\right] \\
\ell_{23}\left(q, \partial_{q}, \lambda\right)=\varepsilon^{-1}[ & \left.\sin \left(\varepsilon q_{2}\right) \partial_{q^{1}}-\tan \left(\varepsilon q_{1}\right) \cos \left(\varepsilon q_{2}\right) \partial_{q^{2}}+\frac{i}{\hbar} \varepsilon j_{2} \sec \left(\varepsilon q_{1}\right) \cos \left(\varepsilon q_{2}\right)\right] .
\end{aligned}
$$


The exponentials of operators $\ell_{1}\left(q, \partial_{q}, \lambda\right), \ell_{2}\left(q, \partial_{q}, \lambda\right)$ and $\ell_{3}\left(q, \partial_{q}, \lambda\right)$ for the fixed $j_{1}=-m$ and $j_{2}=s / 2$ act on a function as follows:

$$
\begin{gathered}
e^{-y \ell_{3}\left(q, \partial_{q}, \lambda\right)} f(q)=f\left(q_{1}, q_{2}-y\right), \\
e^{-x \ell_{2}\left(q, \partial_{q}, \lambda\right)} f(q)=e^{\frac{i s}{2} \operatorname{arccot}\left(\frac{\cot \varepsilon q_{2}}{\sin \varepsilon q_{1}}\right)} \Phi_{2}\left[\cos \varepsilon q_{1} \sin \varepsilon q_{2}, \arctan \left(\frac{\tan \varepsilon q_{1}}{\cos \varepsilon q_{2}}\right)-\varepsilon x\right], \\
\Phi_{2}(a, b)=e^{-\frac{1}{2} i s \arctan (a \tan b)} f\left[\varepsilon^{-1} \arctan \left(\frac{\sqrt{1-a^{2}} \tan b}{\sqrt{a^{2} \tan ^{2} b+1}}\right), \varepsilon^{-1} \operatorname{arcsec}\left(\sqrt{\frac{a^{2} \tan ^{2} b+1}{1-a^{2}}}\right)\right], \\
e^{-t \ell_{1}\left(q, \partial q_{1}, \lambda\right)} f(q)=\frac{\left(\sin \varepsilon q_{1}\right)^{i m / \varepsilon}}{\sqrt{\sin \varepsilon q_{1}\left(\sin \varepsilon q_{1} \cos \varepsilon q_{2}-i \sin \varepsilon q_{2}\right)}} \times \\
\times \Phi_{3}\left[\sin \varepsilon q_{2} \cot \varepsilon q_{1}, \frac{\cos \varepsilon q_{1} \cos \varepsilon q_{2} \sinh \varepsilon t+\cosh \varepsilon t}{\sqrt{1-\left(\cos \varepsilon q_{1} \cos \varepsilon q_{2}\right)^{2}}}\right] \\
\Phi_{3}(a, b)=\left(b \sqrt{a^{2}+1}\right)^{i m / \varepsilon}\left(\frac{\sqrt{b^{2}-1}-i s a b}{b^{2} \sqrt{a^{2}+1} \sqrt{\left(a^{2}+1\right) b^{2}-1}}\right)^{1 / 2} \times \\
f\left[\varepsilon^{-1} \operatorname{arcsec}\left(\frac{\sqrt{a^{2}+1} b}{\sqrt{\left(a^{2}+1\right) b^{2}-1}}\right), \varepsilon^{-1} \arccos \left(\frac{\sqrt{a^{2}+1} \sqrt{b^{2}-1}}{\sqrt{\left(a^{2}+1\right) b^{2}-1}}\right)\right] .
\end{gathered}
$$

\section{References}

1. Birrell, N.; Davies, P. Quantum Fields in Curved Space; Cambridge University Press: Cambrigde, UK, 1986; p. 340

2. Grib, A; Mostepanenko, V; Mamayev, S. Vacuum Quantum Effects in Strong Fields; Fridmann Lab.: St. Petersburg, Russia, 1994; 361p.

3. Ford, L. Vacuum polarization in a nonsimply connected spacetime. Phys. Rev. D 1980, 21, 933. [CrossRef]

4. Frolov, V.P.; Zel'Nikov, A.I. Vacuum polarization of massive fields near rotating black holes. Phys. Rev. D 1984, 29, 1057. [CrossRef]

5. Wald, R.M. The back reaction effect in particle creation in curved spacetime. Commun. Math. Phys. 1977, 54, 1-19. [CrossRef]

6. Kadoyoshi, T.; Sugamoto, A.; Nojiri, S.I.; Odintsov, S.D. Vacuum polarization of supersymmetric D-brane in the constant electromagnetic field. Mod. Phys. Lett. A 1998, 13, 1531-1537. [CrossRef]

7. Kalnins, E. Separation of Variables in Riemannian Spaces of Constant Curvature; Wiley: New York, NY, USA, 1986; 172p.

8. Kalnins, E.G.; Miller, W., Jr.; Williams, G.C. Recent advances in the use of separation of variables methods in general relativity. Philos. Trans. R. Soc. Lond. Ser. A 1992, 340, 337-352.

9. Miller, W. Symmetry and Separation of Variables; Cambridge University Press: Cambridge, UK, 1984; p. 318.

10. Bagrov, V.; Gitman, D. Exact Solutions of Relativistic Wave Equations; Kluwer Academic Publishers: Dordrecht, The Netherlands, 1990; 324p.

11. Stephani, H.; Kramer, D.; MacCallum, M.; Hoenselaers, C.; Herlt, E. Exact Solutions of Einstein's Field Equations, 2nd ed.; Cambridge University Press: Cambridge, UK, 2003; 732p.

12. Ryan, M.; Lawrence, C. Homogeneous Relativistic Cosmologies; Princeton University Press: Princeton, NJ, USA, 2015; 338p.

13. Shapovalov, V.N. Stackel spaces. Sib. Math. J. 1979, 20, 790-800.

14. Shapovalov, V.N. Symmetry and separation of variables in Hamilton-Jacobi equations. I. Sov. Phys. J. 1978, 21, 1124-1129.

15. Obukhov, V. Hamilton-Jacobi Equation for a Charged Test Particle in the Stäckel Space of Type (2.0). Symmetry 2020, 12, 1289. 
16. Shapovalov, A.V.; Shirokov, I.V. non-commutative integration of linear differential equations. Theor. Math. Phys. 1995, 104, 921-934.

17. Shirokov, I.V. Symmetry in Nonlinear Mathematical Physics. Proc. Inst. Math. NAS Ukr. 2003, 50, $246-251$.

18. Baranovskii, S.P.; Mikheev, V.V.; Shirokov, I.V. Quantum Hamiltonian systems on K-orbits: semiclassical spectrum of the asymmetric top. Theor. Math. Phys. 2001, 129, 1311-1319.

19. Breev, A.I.; Magazev, A.A.; Shirokov, I.V. Vacuum polarization of a scalar field on Lie groups and homogeneous spaces. Theor. Math. Phys. 2011, 167, 468-483. [CrossRef]

20. Breev, A.I. Scalar field vacuum polarization on homogeneous spaces with an invariant metric. Theor. Math. Phys. 2014, 178, 59-75. [CrossRef]

21. Breev, A.I.; Kozlov, A.V. Vacuum Averages of the Energy-Momentum Tensor of a Scalar Field in Homogeneous Spaces with a Conformal Metric. Russ. Phys. J. 2016,58, 1248-1257. [CrossRef]

22. Fedoseev, V.G.; Shapovalov, A.V.; Shirokov, I.V. On non-commutative solution of the Dirac equation in Riemann space with a dynamical group. Izv. Vuz. Phys. 1991, 34, 43-46.

23. Shapovalov, A.V.; Shirokov, I.V. non-commutative integration of Klein-Gordon and Dirac equations with movement group. Izv. Vuz. Phys. 1991, 34, 33-38.

24. Varaksin, O.L.; Shirokov, I.V. Integration of the Dirac equation, which does not presume complete separation of variables, in Stäckel spaces. Russ. Phys. J. 1996, 39, 27-32. [CrossRef]

25. Varaksin, O.L.; Klishevich, V.V. Integration of Dirac equation in Riemannian spaces with five-dimensional group of motions. Russ. Phys. J. 1997, 40, 727-731. [CrossRef]

26. Klishevich, V.V. Integration of the Dirac equation in Riemannian space with group of motions. I. Russ. Phys. J. 2000, 43, 1038-1043.

27. Klishevich, V.V. Exact solution of Dirac and Klein-Gordon-Fock equations in a curved space admitting a second Dirac operator. Class. Quantum Gravity 2001, 18, 3735. [CrossRef]

28. Tyumentsev, V.A.; Klishevich, V.V. non-commutative Integration of the Dirac Equation in a Flat Space and in the de Sitter Space. Russ. Phys. J. 2003, 46, 891-896.

29. Klishevich, V.V.; Tyumentsev, V.A. On the solution of the Dirac equation in de Sitter space. Class. Quantum Gravity 2005, 22, 4263. [CrossRef]

30. Breev, A.I.; Shirokov, I.V. Polarization of a spinor field vacuum on manifolds of the Lie groups Russ. Phys. J. 2009, 52, 823-832.

31. Breev, A.I.; Magazev, A.A. Integration of the Dirac equation on Lie groups in an external electromagnetic field admitting a non-commutative symmetry algebra. Russ. Phys. J. 2017, 59, 2048-2058.

32. Dubrovin, B.; Fomenko, A.; Novikov, S. Modern Geometry and Applications, Part III: Introduction to Homology Theory; Graduate Texts in Mathematics; Springer: New York, NY, USA, 1990; 418p.

33. Kobayashi, S.; Nomizu, K. Foundations of Differential Geometry; Wiley-Interscience: New York, NY, USA, 1996; Volume I; 348p.

34. Arvanitogeōrgos, A. An Introduction to Lie Groups and the Geometry of Homogeneous Spaces; Student Mathematical Library V. 22; American Mathematical Society: Providence, RI, USA, 2003; 141p.

35. Kurnyavko, O.L.; Shirokov, I.V. Construction of invariant scalar particle wave equations on Riemannian manifolds with external gauge fields. Theor. Math. Phys. 2008, 156, 1169-1179. [CrossRef]

36. Baranovskiii, S.P.; Shirokov, I.V. Prolongations of vector fields on Lie groups and homogeneous spaces. Theor. Math. Phys. 2003, 135, 510-519.

37. Kirillov, A. Lectures on the Orbit Method; Graduate Studies in Mathematics V. 64; American Mathematical Society: Providence, RI, USA, 2004; 408p.

38. Magazev, A.A.; Mikheyev, V.V.; Shirokov, I.V. Computation of composition functions and invariant vector fields in terms of structure constants of associated Lie algebras. SIGMA 2015, 11, 066. [CrossRef]

39. Shirokov, I.V. Darboux coordinates on K-orbits and the spectra of Casimir operators on Lie groups. Theor. Math. Phys. 2000, 123, 754-767. [CrossRef]

40. Shirokov, I.V. Identities and invariant operators on homogeneous spaces. Theor. Math. Phys. 2001, 126, $326-338$.

41. Dixmier, J. Enveloping Algebras; North-Holland Mathematical Studies; Elsevier: Amsterdam, The Netherlands, 1977; Volume 14, 376p.

42. Barut, A; Raczka, R. Theory of Group Representations and Applications, 2nd ed.; World Scientific Publishing Company: London, UK, 1986; 740p. 
43. Bagrov, V.G.; Shapovalov, A.V.; Yevseyevich, A.A. Separation of variables in the Dirac equation in Stackel spaces. II. External gauge fields. Class. Quantum Gravity 1991, 8, 163-173. [CrossRef]

44. Breev, A.I.; Goncharovskii, M.M.; Shirokov, I.V. Klein-Gordon equation with a special type of nonlocal nonlinearity in commutative homogeneous spaces with invariant metric. Russ. Phys. J. 2013, 56, 731-739. [CrossRef]

45. Hack, T. Cosmological Applications of Algebraic Quantum Field Theory in Curved Spacetimes, 1st ed.; SpringerBriefs in Mathematical Physics V. 6; Springer: New York, NY, USA, 2016; 131p.

46. Toms, D.J. Effective action for the Yukawa model in curved spacetime. J. High Energy Phys. 2018, 5, 139. [CrossRef]

47. Nojiri, S.I.; Odintsov, S.D. Effective equation of state and energy conditions in phantom/tachyon inflationary cosmology perturbed by quantum effects. Phys. Lett. B 2003, 571, 1-10. [CrossRef]

48. Brevik, I.; Milton, K.A.; Odintsov, S.D.; Osetrin, K.E. Dynamical Casimir effect and quantum cosmology. Phys. Rev. D 2000, 62, 064005. [CrossRef]

49. Vafek, O.; Vishwanath, A. Dirac Fermions in Solids-from High Tc cuprates and Graphene to Topological Insulators and Weyl Semimetals. ARCMP 2014, 5, 83-112.

50. Klimchitskaya, G.L.; Mostepanenko, V.M. Creation of quasiparticles in graphene by a time-dependent electric feld. Phys. Rev. D 2013, 87, 125011. [CrossRef]

51. Antoniadis, I.; Arkani-Hamed, N.; Dimopoulos, S.; Dvali, G. New dimensions at a millimeter to a Fermi and superstrings at a TeV. Phys. Lett. B 1998, 436, 257-263. [CrossRef]

52. Perelomov, A. Generalized Coherent States and Their Applications; Theoretical and Mathematical Physics; Springer: Dordrecht, The Netherlands, 2012; 418p.

53. Malkin, I.; Manko, V. Dynamic Symmetry and Coherent States of Quantum Systems; Nauka: Moscow, Nauka, 1979.

54. Perelomov, A.M. Coherent states for arbitrary Lie group. Commun. Math. Phys. 1972, 26, 222-236. [CrossRef]

55. Bagrov, V.; Gitman, D. The Dirac Equation and Its Solutions; Walter de Gruyter GmbH: Brelin/Boston, Germany, $2014 ; 444$ p.

Publisher's Note: MDPI stays neutral with regard to jurisdictional claims in published maps and institutional affiliations.

(C) 2020 by the authors. Licensee MDPI, Basel, Switzerland. This article is an open access article distributed under the terms and conditions of the Creative Commons Attribution (CC BY) license (http:/ / creativecommons.org/licenses/by/4.0/). 